\title{
Finding Topological Subgraphs is Fixed-Parameter Tractable
}

\author{
Martin Grohe \\ Institut für Informatik \\ Humboldt-Universität zu Berlin \\ Unter den Linden 6 \\ 10099 Berlin, Germany \\ grohe@informatik.hu- \\ berlin.de \\ Dániel Marx \\ Institut für Informatik \\ Humboldt-Universität zu Berlin \\ Unter den Linden 6 \\ 10099 Berlin, Germany \\ dmarx@informatik.hu- \\ berlin.de
}

\begin{abstract}
We prove that for every fixed undirected graph $H$, there is an $O\left(|V(G)|^{3}\right)$ time algorithm that, given a graph $G$, tests if $G$ contains $H$ as a topological subgraph (that is, a subdivision of $H$ is subgraph of $G$ ). This shows that topological subgraph testing is fixed-parameter tractable, resolving a longstanding open question of Downey and Fellows from 1992. As a corollary, for every $H$ we obtain an $O\left(|V(G)|^{3}\right)$ time algorithm that tests if there is an immersion of $H$ into a given graph $G$. This answers another open question raised by Downey and Fellows in 1992.
\end{abstract}

\section{Categories and Subject Descriptors}

F.2 [Theory of Computing]: Analysis of Algorithms and Problem Complexity

\section{General Terms}

Algorithms

\section{Keywords}

topological minors, fixed-parameter tractability

\footnotetext{
*Research partly supported by Japan Society for the Promotion of Science, Grant-in-Aid for Scientific Research, by Kayamori Foundation and by Inoue Research Award for Young Scientists.

${ }^{\dagger}$ Research supported in part by ERC Advanced grant DMMCA, the Alexander von Humboldt Foundation, and the Hungarian National Research Fund (Grant Number OTKA 67651).
}

Permission to make digital or hard copies of all or part of this work for personal or classroom use is granted without fee provided that copies are not made or distributed for profit or commercial advantage and that copies bear this notice and the full citation on the first page. To copy otherwise, to republish, to post on servers or to redistribute to lists, requires prior specific permission and/or a fee.

STOC'11, June 6-8, 2011, San Jose, California, USA

Copyright 2011 ACM 978-1-4503-0691-1/11/06 ...\$10.00.

\author{
Ken-ichi Kawarabayashi* \\ National Institute of \\ Informatics \\ 2-1-2 Hitotsubashi, \\ Chiyoda-ku \\ Tokyo 101-8430, Japan \\ k_keniti@nii.ac.jp \\ Paul Wollan \\ Department of Computer \\ Science \\ University of Rome, La Sapienza \\ Via Salaria 113 \\ Rome, 00198 Italy \\ wollan@di.uniroma1.it
}

\section{Introduction}

A graph $H$ is a topological subgraph (or topological minor) of graph $G$ if a subdivision of $H$ is a subgraph of $G$. Equivalently, $H$ is a topological subgraph of $G$ if $H$ can be obtained from $G$ by deleting edges, deleting vertices, and dissolving degree 2 vertices (which means deleting the vertex and making its two neighbors adjacent). This notion appears for example in the classical result of Kuratowski in 1935 stating that a graph is planar if and only if it does not have a topological subgraph isomorphic to $K_{5}$ or $K_{3,3}$.

Given graphs $H$ and $G$, it is NP-complete to decide if $H$ is a topological subgraph of $G$ (e.g., a cycle of length $|V(G)|$ is a topological subgraph of $G$ if and only if $G$ is Hamiltonian). On the other hand, our main result gives a cubic algorithm for every fixed $H$ :

THEOREM 1.1. For every fixed undirected graph $H$, there is a $O\left(|V(G)|^{3}\right)$ time algorithm that decides if $H$ is a topological subgraph of $G$.

Actually, our algorithm is uniform in $H$, and this shows that the problem of testing if $H$ is a topological subgraph of $G$ is fixedparameter tractable parameterized by the number of vertices of $H$. Recall that a problem is fixed-parameter tractable by some parameter $k$ if it can be solved in time $f(k) \cdot n^{O(1)}$ for a function $f$ depending only on $k$. Thus Theorem 1.1 answers a longstanding open question, first raised in 1992 by Downey and Fellows [3] and then restated at many places, including the open problem list of the monograph [4]. The problem of testing for topological subgraphs, which is also known as the subgraph homeomorphism problem, was already studied in the 1970s by Lapaugh and Rivest [10] (also see [7]). Fortune, Hopcroft, and Wyllie [6] studied the directed version of the problem and showed that there are simple digraphs $H$ such that the problem of testing whether a given digraph $G$ contains $H$ as a (directed) topological subgraph is NP-complete. In a major breakthrough, Robertson and Seymour [11] proved that this cannot happen for undirected graphs: For every (undirected) graph $H$ there is a polynomial time algorithm testing whether a given graph $G$ contains $H$ as a topological subgraph. (We will discuss Robertson and Seymour's result in more detail below.) However, the running time of Robertson and Seymour's algorithm is $|V(G)|^{|V(H)|}$. This 
prompted Downey and Fellows' questions of whether the problem is fixed-parameter tractable. Our Theorem 1 answers this question.

We also study the related problem of testing for immersed subgraphs. An immersion of a graph $H$ into a graph $G$ is defined like a topological embedding, expect that the paths in $G$ corresponding to the edges of $H$ are only required to be edge disjoint instead of internally vertex disjoint. Formally, an immersion of $H$ into $G$ is a mapping $\alpha$ that associates with each vertex $v \in V(H)$ a distinct vertex $\alpha(v) \in V(G)$ and with each edge $e=v w \in E(H)$ a path $\alpha(e)$ in $G$ with endpoints $\alpha(v)$ and $\alpha(w)$ in such a way that the paths $\alpha(e)$ for $e \in E(H)$ are mutually edge disjoint. Robertson and Seymour [14] showed that graphs are well-quasi-ordered under the immersion relation, proving a conjecture of Nash-Williams. Here we obtain the following algorithmic result as a corollary to Theorem 1.1:

COROLlARY 1.2. For every fixed undirected graph $H$, there is a $O\left(|V(G)|^{3}\right)$ time algorithm that decides if there is an immersion of $H$ into $G$.

Again, our algorithm is uniform in $H$, which implies that the immersion problem is fixed-parameter tractable. This answers another open question by Downey and Fellows [3, 4]. Corollary 1.2 also holds for the more restrictive "strong immersion" version, where $\alpha(v)$ cannot be the internal vertex of $\alpha(e)$ for any $v \in V(G)$ and $e \in E(G)$.

Yet another related problem is minor containment testing. We say that graph $H$ is a minor of $G$ if $H$ can be obtained from $G$ by deleting vertices, deleting edges, and contracting edges. A celebrated result of Robertson and Seymour [11] shows that for every fixed $H$, there is a $O\left(|V(G)|^{3}\right)$ time algorithm for testing if $H$ is a minor of $G$. Their algorithm actually solves a more general rooted version of the problem. This rooted version contains as a special case the $k$-DISJOINT PATHS problem, where given pairs $\left(s_{1}, t_{1}\right)$, $\ldots,\left(s_{k}, t_{k}\right)$ of vertices, the task is to find vertex disjoint paths $P_{1}$, $\ldots, P_{k}$ such that $P_{i}$ connects $s_{i}$ and $t_{i}$. It is not difficult to reduce testing if $H$ is a topological subgraph of $G$ to $k$-DisJOINT PATHS. For each vertex $v$ of $H$, we guess a vertex $v^{\prime}$ of $G$, and then for each edge $u v$ of $H$, we find a path connecting $u^{\prime}$ and $v^{\prime}$ in $G$ such that these $|E(H)|$ paths are pairwise internally disjoint. This approach yields the $|V(G)|^{O(|V(H)|)}$ time algorithm for topological subgraph testing mentioned above.

Our algorithm for finding topological subgraphs follows the general framework of Robertson and Seymour for minor testing, but it deviates from it significantly. Let us give a very high-level overview of Robertson and Seymour's algorithm [11]. If the treewidth of $G$ is "small," then standard techniques allow us to solve the problem in linear time. If the treewidth of $G$ is "large," then we find an irrelevant vertex whose deletion provably does not change the answer to the problem. By iteratively finding and deleting irrelevant vertices, we eventually arrive to a $G$ whose treewidth is small. To find an irrelevant vertex if the treewidth of $G$ is large, we use the the so-called Weak Structure Theorem, which allows us either to find a large clique minor or to show that the graph has a large "flat wall." The case of a large clique minor is easy to handle: if there are no roots, then it immediately solves the problem (as every small graph appears in the large clique minor) and even if roots are present, we can argue that a large part of the clique is irrelevant. The most difficult part of the algorithm is to deal with the case of a flat wall and to identify an irrelevant vertex there. Indeed, this case needs the majority of the work. The analysis of this case requires the whole series of Graph Minor papers and the structure theorem of [12]. Very recently, a significantly simpler treatment of this case was presented in [9].
Let us now give an overview for our algorithm. The case of small treewidth goes through for topological minor testing without any difficulty. The new proof in [9] for minor testing in the case when there is no large clique minor can be adapted for topological minor testing. Specifically, for the case where there is a large flat wall, using the unique linkage theorem [13] and its much shorter proof [9], we can indeed find an irrelevant vertex in the middle of the large flat wall. This case is similar to that for the minor testing, however, we may need to change almost all of the branch vertices of a given topological minor inside the flat wall. This gives rise to some amount of technical difficulties, which we overcome in this paper. Let us emphasis that our proof of the correctness for our algorithm does not depend on the full power of the graph minor structure theorem [12], while Robertson and Seymour's analysis for their algorithm does need the whole series of Graph Minor papers and the structure theorem of [12]. Utilizing some results in [9], we are able to avoid the much of the heavy machinery of the graph minor structure theory.

Let us now look at the case when there is a large clique minor. Identifying a large clique minor was an easy situation to handle in the case of finding minors, but it is not obvious how it is of any use in the case of finding topological subgraphs. The problem is that the degrees of the vertices matter much more in finding topological subgraphs than in finding minors. If $H$ is, say, 4-regular and we have found a large clique minor in a part of $G$ that contains only degree- 3 vertices, then this clique minor does not immediately solve the problem. Furthermore, as $G$ can contain many vertices of degree at least 4 close to this clique minor and each such vertex is potentially the image of some vertex of $H$, there is no easy argument that shows that some part of the clique is irrelevant. We circumvent these problems by introducing a new operation that was not present in the framework of [11]. If a small number of vertices can separate away a large part of the graph, then we recursively "understand" this part and then replace it with an equivalent smaller graph. We show that if no such step can be performed, then we can completely understand how the large clique minor can be used by a topological subgraph. This new operation and the associated recursion changes the high-level structure of our algorithm considerably: unlike in [11], it is no longer just an iterative removal of irrelevant vertices.

Similarly to [11], we define and solve a very general rooted version of the problem ("finding folios"). It is important to point out that we are solving this rooted generalization not (only) for the sake of obtaining maximum generality of the result. In the recursion steps involving separators, we argue about topological subgraphs using the separator in a certain way, and the concept of roots is needed to express these requirements.

Due to the page limitations of this extended abstract, we discuss in detail only the main algorithmic framework (Section 3 ), the case of handling a large clique minor (Section 4), and the reduction from immersion testing to finding topological subgraphs. These are the parts that contain the most significant differences compared to minor testing. While the case of handling a large flat wall also required overcoming significant technical difficulties, the treatment of this case appears in the full version.

\section{Folios}

All graphs in this paper are finite and simple: they do not have loops or parallel edges (but can have isolated vertices). A rooted graph is an undirected graph $G$ with a set $R(G) \subseteq V(G)$ of vertices specified as roots and an injective mapping $\rho_{G}: R(G) \rightarrow \mathbb{N}$ assigning a distinct positive integer label to each root vertex. Isomor- 
phism of rooted graphs are defined the obvious way, i.e., roots must be mapped to roots with the same label. We say that two rooted graphs $G_{1}$ and $G_{2}$ are compatible if $\rho_{G_{1}}\left(R\left(G_{1}\right)\right)=\rho_{G_{2}}\left(R\left(G_{2}\right)\right)$, i.e. the same set of positive integers appear on $G_{1}$ and $G_{2}$ (which means in particular that $\left|R\left(G_{1}\right)\right|=\left|R\left(G_{2}\right)\right|$ ).

We say that rooted graph $H$ is a topological minor of rooted graph $G$ if there is a mapping $\phi$ (a model of $H$ in $G$ ) that assigns to each $v \in V(H)$ a vertex $\phi(v) \in V(G)$ and to each $e \in E(G)$ a path $\phi(e)$ in $G$ such that

(1) The vertices $\phi(v)(v \in V(H))$ are distinct.

(2) If $u, v \in V(H)$ are the endpoints of $e \in E(H)$, then path $\phi(e)$ connects $\phi(u)$ and $\phi(v)$.

(3) The paths $\phi(e)(e \in E(H))$ are pairwise internally vertex disjoint, i.e., the internal vertices of $\phi(e)$ do not appear as an (internal or end) vertex of $\phi\left(e^{\prime}\right)$ for any $e^{\prime} \neq e$.

(4) For every $v \in R(H), \rho_{G}(\phi(v))=\rho_{H}(v)$.

Even if $H$ is a topological minor of $G$, they are not necessarily compatible: $G$ can have more root vertices than $H$.

The folio of $G$ is the set of all topological minors of $G$. Clearly, the folio is closed under isomorphism, i.e., if rooted graphs $H$ and $H^{\prime}$ are isomorphic and $H$ is in the folio of $G$, then $H^{\prime}$ is in the folio as well. If $\delta \geq 0$ is an integer, then the $\delta$-folio of $G$ contains every topological minor $H$ of $G$ with $|E(H)|+$ is $(H) \leq \delta$, where is $(H)$ is the number of isolated vertices of $H$. Obviously, every graph in the $\delta$-folio has at most $2 \delta$ vertices.

OBSERVATION 2.1. The number of distinct graphs (up to isomorphism) in the $\delta$-folio of $G$ can be bounded by a function of $\delta$ and $|R(G)|$.

There are $2\left(\begin{array}{c}(R(G) \mid \\ 2\end{array}\right)$ possible undirected graphs on $R(G)$. For each such graph $X$, we slightly abuse notation by defining $G+X$ to be the graph on $V(G)$ having edge set $E(G) \cup E(X)$. The rooted graph $G+X$ has a $\delta$-folio, which may or may not be different from the $\delta$ folio of $G$. The $2\left(\begin{array}{c}|R(G)| \\ 2\end{array}\right)$-tuple of all these $\delta$-folios will be called the extended $\delta$-folio of $G$. To extended $\delta$-folios are considered equal if the folios are equal for each choice of the set $X$.

Given an extended $\delta$-folio $\mathcal{F}$, a representative of $\mathcal{F}$ is a rooted graph $G$ whose extended $\delta$-folio is $\mathcal{F}$. We define the constant $L_{\delta, r}$ to be the smallest integer such that for every rooted graph $G$ with at most $r$ roots, the extended $\delta$-folio of $G$ has a representative on at most $L_{\delta, r}$ vertices. It is clear that $L_{\delta, r}$ is finite.

LEMMA 2.2. There is a computable function $\ell(\delta, r)$ with $L_{\delta, r} \leq$ $\ell(\delta, r)$ for every $\delta, r \geq 0$.

The (extended) $\delta$-folio of a graph $G$ with respect to a set $Z \subseteq$ $V(G)$ is the (extended) $\delta$-folio of the graph $G^{\prime}$, where $G^{\prime}$ has the same set of vertices and edges as $G$, but $R\left(G^{\prime}\right)=Z$. We will use this notion to avoid defining new graphs that differ only in the set of roots. Some straightforward observations:

Proposition 2.3. Let $G$ be a rooted graph and let $\delta \geq 0$ be an integer.

(1) The extended 0 -folio of $G$ contains only the empty graph.

(2) Let $R \subseteq Q \subseteq V(G)$ be two sets of vertices. The $\delta$-folio of $G$ with respect to $R$ can be computed from the $\delta$-folio of $G$ with respect to $Q$.

(3) Let $R_{1}, \ldots, R_{t}$ be subsets of $V(G)$ such that for every subset $Q \subseteq R(G)$ of size at most $2 \delta$ there is a $1 \leq i \leq t$ such that $Q \subseteq R_{i}$. The $\delta$-folio of $G$ can be computed from the $\delta$-folios of $G$ with respect to $R_{1}, \ldots, R_{t}$.

(4) The extended $\delta$-folio of $G$ can be computed from the $(\delta+$ $|R(G)|)$-folio of $G$.

\subsection{Separations and replacements}

A separation of a graph $G$ is a pair $(A, B)$ of subgraphs such that $V(G)=V(A) \cup V(B), E(G)=E(A) \cup E(B)$, and $E(A) \cap E(B)=\emptyset$. The order of the separation $(A, B)$ is $|V(A) \cap V(B)|$.

Let $(A, B)$ be a separation of rooted graph $G$ such that $V(A) \cap$ $V(B) \subseteq R(G)$. Let $A^{\prime}$ be a rooted graph compatible with $A$. Replacing $A$ with $A^{\prime}$ in the separation $(A, B)$ gives the graph $G^{\prime}$ defined as follows. We have $V\left(G^{\prime}\right)=V\left(A^{\prime}\right) \cup(V(B) \backslash V(A)), G^{\prime}$ has every edge of $A^{\prime}$ and $B \backslash V(A)$, and $G^{\prime}$ has the following additional edges: if $u \in V(A) \cap V(B)$ and $v \in V(B) \backslash V(A)$ are adjacent in $G$, and $u^{\prime} \in V\left(A^{\prime}\right)$ is a vertex with $\rho_{A}(u)=\rho_{A^{\prime}}\left(u^{\prime}\right)$, then $u^{\prime}$ and $v$ are adjacent in $G^{\prime}$. Intuitively, we remove $A$ from $G$, and replace it by $A^{\prime}$ such that the role of $V(A) \cap V(B)$ is taken by the matching root vertices of $A^{\prime}$. The following lemmas show how the folio changes after replacement:

LEMMA 2.4. Let $\left(G_{1}, G_{2}\right)$ be a separation of a rooted graph $G$, let $S=V\left(G_{1}\right) \cap V\left(G_{2}\right)$, and suppose that $S \subseteq R(G)$. Let $G_{1}^{\prime}$ be a rooted graph compatible with $G_{1}$ such that $G_{1}$ and $G_{1}^{\prime}$ have the same extended $\delta$-folio. Let $G^{\prime}$ be the graph obtained by replacing $G_{1}$ with $G_{1}^{\prime}$ in the separation $\left(G_{1}, G_{2}\right)$. Then $G$ and $G^{\prime}$ have the same extended $\delta$-folio.

PROOF. Without loss of generality, we can assume that $R(G) \cap$ $V\left(G_{1}\right)=S$ : extending $G_{2}$ such that $V\left(G_{2}\right)$ fully contains $R(G)$ does not change the statement of the theorem. Under this assumption, it is sufficient to prove the weaker statement that $G$ and $G^{\prime}$ have the same (not extended) $\delta$-folio (but the condition that $G_{1}$ and $G_{1}^{\prime}$ have the same extended $\delta$-folio is not changed). To see this, consider an arbitrary graph $X$ on $R(G)$. Let $X_{1}$ be the subgraph of $X$ induced by $R(G) \cap V\left(G_{1}\right)=S$ and let $X_{2}=X \backslash E\left(X_{1}\right)$. Now $G+X$ has a separation $\left(G_{1}+X_{1}, G_{2}+X_{2}\right)$ and $G^{\prime}+X$ has a separation $\left(G_{1}^{\prime}+X_{1}, G_{2}^{\prime}+X_{2}\right)$. As $G_{1}$ and $G_{1}^{\prime}$ have the same extended $\delta$-folio, graphs $G_{1}+X_{1}$ and $G_{1}^{\prime}+X_{1}$ have the same extended $\delta$-folio as well. Therefore, the weaker statement shows that $G+X$ and $G^{\prime}+X$ have the same $\delta$-folio. As this is true for every $X$ on $R(G)$, it follows that $G$ and $G^{\prime}$ have the same extended $\delta$-folio.

Let $H$ be a rooted graph with $|E(H)|+$ is $(H) \leq \delta$ and let $\phi$ be a model of $H$ in $G$. We need to show that $H$ has a model $\phi^{\prime}$ in $G^{\prime}$.

We define the graph $X^{*}$ on $S=R(G) \cap V\left(G_{1}\right)$ such that $u v \in$ $X^{*}$ for some $u, v \in S$ if there is an edge $e \in E(H)$ such that $\phi(e)$ has a subpath with endpoints $u$ and $v$ and every internal vertex in $V\left(G_{2}\right) \backslash V\left(G_{1}\right)$. For every $u v \in E\left(X^{*}\right)$, let $P_{u v}$ be this subpath. Given a path $P$ in $G$ with endpoints in $V\left(G_{1}\right)$, we denote by $[P]_{G_{1}}$ the path obtained by replacing subpaths of $P$ that leave $V\left(G_{1}\right)$ by appropriate edges of $X^{*}$. Similarly, if $Q$ is a path in $G_{1}+X^{*}$, then we denote by $[Q]^{G}$ the path of $G$ obtained by replacing each edge $u v$ of $X^{*}$ by the corresponding path $P_{u v}$.

We define a graph $H^{*}$ and a model $\psi$ of $H^{*}$ in $G_{1}+X^{*}$ as follows. First, graph $H^{*}$ contains every vertex $v \in V(H)$ with $\phi(v) \in$ $V\left(G_{1}\right)$; if $v \in R(H)$, then $v$ is in $R\left(H^{*}\right)$ and has the same root number in $H$ and $H^{*}$. For such vertices, we set $\psi(v)=\phi(v)$. We introduce additional vertices and edges to $H^{*}$ as follows. We classify each edge $e=u v \in V(H)$ into one of 6 types, and modify $H^{*}$ accordingly.

(1) $\phi(u), \phi(v) \in V\left(G_{1}\right)$. For each such edge, there is a corresponding edge $e^{*}=u v$ in $H^{*}$. We define $\psi\left(e^{*}\right)=[\phi(e)]_{G_{1}}$.

(2) $\phi(u) \in V\left(G_{1}\right), \phi(v) \notin V\left(G_{1}\right)$, and $\phi(e)$ has an internal vertex in $V\left(G_{1}\right)$. For each such edge, let us introduce a new vertex $v_{e}^{*}$ that has the same root number as the last vertex $w$ of $\phi(e)$ (going from $u$ to $v$ ) that is in $V\left(G_{1}\right)$. Note that this last vertex has to be in $S \subseteq R(G)$, hence it is a root vertex. Let $\psi\left(v_{e}^{*}\right)=$ $w$. We introduce an edge $e^{*}=u v_{e}^{*}$ in $H^{*}$ and set $\psi\left(e^{*}\right)=$ $[P]_{G_{1}}$, where $P$ is the subpath of $\phi(e)$ from $u$ to $w$. 
(3) $\phi(u) \in V\left(G_{1}\right), \phi(v) \notin V\left(G_{1}\right)$, and $\phi(e)$ has no internal vertex in $V\left(G_{1}\right)$. This is only possible if $u \in V\left(G_{1}\right) \cap V\left(G_{2}\right)$, hence $u$ is a root. We modify $H^{*}$ by making $u$ a root (if it is not already a root), having the same root number as $\phi(u)$.

(4) $\phi(u), \phi(v) \notin V\left(G_{1}\right)$, and $\phi(e)$ has no internal vertex in $V\left(G_{1}\right)$. No change is done to $H^{*}$.

(5) $\phi(u), \phi(v) \notin V\left(G_{1}\right)$, and $\phi(e)$ has a single internal vertex $w$ in $V\left(G_{1}\right)$. This is only possible if $w \in V\left(G_{1}\right) \cap V\left(G_{2}\right)$, and hence $w$ is a root. An isolated root vertex $i_{e}^{*}$ is introduced to $H^{*}$, with the same root number as $w$. Let $\psi\left(i_{e}^{*}\right)=w$.

(6) $\phi(u), \phi(v) \notin V\left(G_{1}\right)$, and $\phi(e)$ has more than one internal vertex in $V\left(G_{1}\right)$. Let $u_{e} \neq v_{e}$ be the first and last vertices, respectively, on $\phi(e)$ (going from $u$ to $v$ ) that are in $V\left(G_{1}\right)$. Note that $u_{e}$ and $v_{e}$ are in $V\left(G_{1}\right) \cap V\left(G_{2}\right)$, hence they are root vertices. Let us introduce root vertices $v_{e}^{*}$ and $u_{e}^{*}$ in $H^{*}$ that have the same root numbers as $u_{e}$ and $v_{e}$, respectively; let $\psi\left(u_{e}^{*}\right)=u_{e}$ and $\psi\left(v_{e}^{*}\right)=v_{e}$. Let us also introduce an edge $e^{*}$ connecting $v_{e}^{*}$ and $u_{e}^{*}$, and let $\psi\left(e^{*}\right)=[P]_{G_{1}}$, where $P$ is the subpath of $\phi(e)$ from $u_{e}$ to $v_{e}$.

This completes the description of $H^{*}$. It should be clear that $\psi$ is a model of $H^{*}$ in $G_{1}+X^{*}$. Furthermore, we claim that $\left|E\left(H^{*}\right)\right|+$ is $\left(H^{*}\right) \leq|E(H)|+$ is $(H) \leq \delta$. First, for each edge of $H$, we introduce at most one edge in $H^{*}$ (for type 3-5 edges, we introduce no new edge in $H^{*}$ ). Moreover, a vertex of $H^{*}$ can be isolated only if it was isolated in $H$, or only type 3 edges were adjacent to it, or it was introduced introduced as a vertex $i_{e}^{*}$ corresponding to a type 5 edge $e$. This means that the number of isolated vertices in $H^{*}$ is at most is $(H)$ plus the number of type 3-5 edges in $H$.

As $H^{*}$ is a topological minor of $G_{1}+X^{*}$, it is a topological minor of $G_{1}^{\prime}+X^{*}$ as well; let $\psi^{\prime}$ be a model of $H^{*}$ in $G_{1}^{\prime}+X^{*}$. We show that $\psi^{\prime}$ can be used to define a model $\phi^{\prime}$ of $H$ in $G^{\prime}$, what we need to show. For every $v \in V(H)$ with $\phi(v) \in V\left(G_{1}\right)$, let $\phi^{\prime}(v)=\psi^{\prime}(v)$ (as $v \in V\left(H^{*}\right)$ in this case) and for every $v \in V(H)$ with $\phi(v) \in$ $V\left(G_{2}\right) \backslash V\left(G_{1}\right)$, let $\phi^{\prime}(v)=\phi(v)$. The images of the 6 different type of edges in $H$ are defined as follows.

(1) Let $\phi^{\prime}(e):=\left[\psi^{\prime}(e)\right]^{G^{\prime}}$.

(2) Let $w \in S$ be the last vertex on $\phi(e)$ from $u$ to $v$. We obtain $\phi^{\prime}(e)$ by concatenating $\left[\psi^{\prime}\left(u v_{e}^{*}\right)\right]^{G^{\prime}}$ (which goes from $\psi^{\prime}(u)$ to $w)$ and the subpath of $\phi(e)$ from $w$ to $v$.

(3) $\phi^{\prime}(e):=\phi(e)$.

(4) $\phi^{\prime}(e):=\phi(e)$.

(5) $\phi^{\prime}(e):=\phi(e)$.

(6) The path $\phi^{\prime}(e)$ is obtained by concatenating the subpath of $\phi(e)$ from $u$ to $u_{v}$, the path $\left[\psi^{\prime}\left(u_{e}^{*} v_{e}^{*}\right)\right]^{G^{\prime}}$, and the subpath of $\phi(e)$ from $u_{v}$ to $u$.

It is not difficult to verify that the paths $\phi^{\prime}(e)$ defined above are internally disjoint. What is important to observe is that if a subpath of $\phi(e)$ is used in the definition above, then every vertex of this subpath in $V\left(G_{1}\right) \cap V\left(G_{2}\right)$ corresponds to a root of $H^{*}$, hence it cannot conflict with that paths $\psi^{\prime}(e)$. Thus $\phi^{\prime}$ is a model of $H$ in $G^{\prime}$, what we had to show.

Lemma 2.4 implies that a separation allows us to determine the folio from the folios of two smaller graphs.

Proposition 2.5. Let $\left(G_{1}, G_{2}\right)$ be a separation of a rooted graph $G$, let $S=V\left(G_{1}\right) \cap V\left(G_{2}\right)$, and suppose that $S \subseteq R(G)$. The extended $\delta$-folio of $G$ can be computed from the extended $\delta$-folios of $G_{1}$ and $G_{2}$.
Given a rooted graph $G$, let $w$ be a weight function that assigns a positive integer to each vertex of $V(G)$. The $w$-bounded $\delta$-folio of $G$ contains those members $H$ of the $\delta$-folio of $G$ that have a model $\phi$ satisfying the additional requirement that for every $v \in R(H)$, the degree of $v$ in $H$ is at most $w(\phi(v))$. Note that we do not make any restriction on the degree of a non-root vertex $u$ of $H$, even if $\phi(u)$ happens to be a root vertex of $G$. The term unbounded $\delta$-folio is used when we want to emphasize that we are referring to the original definition of $\delta$-folio. The $w$-bounded extended $\delta$-folio is defined analogously. Given a weight function $w$ on the vertices of $G$, we define $w(S)=\sum_{v \in S} w(v)$ for every $S \subseteq V(G)$.

Lemma 2.4 does not remain true for $w$-bounded folios: it is not true that $G$ and $G^{\prime}$ have the same $w$-bounded extended $\delta$-folio if $G_{1}$ and $G_{1}^{\prime}$ have the same $w$-bounded extended $\delta$-folio. The particular point where the proof would fail is that a type 3 edge can make a vertex of $H$ a root which was not a root in $H$, and therefore it is not true that the model $\psi$ is $w$-bounded. However, the proof can be fixed if we impose the additional assumption that $G_{1}$ and $G_{1}^{\prime}$ have the same unbounded extended $(\delta-1)$-folio. This statement will be used in Section 4 in a situation where the $w$-bounded $\delta$-folio of $G_{1}$ is easy to determine and we can use recursion to compute the unbounded $(\delta-1)$-folio.

LEMMA 2.6. Let $\left(G_{1}, G_{2}\right)$ be a separation of a rooted graph $G$, let $S=V\left(G_{1}\right) \cap V\left(G_{2}\right)$, and suppose that $S \subseteq R(G)$. Let $w$ be a weight function that assigns a positive integer to each vertex of $V(G)$. Let $G_{1}^{\prime}$ be a rooted graph compatible with $G_{1}$ such that $G_{1}$ and $G_{1}^{\prime}$ have the same $w$-bounded extended $\delta$-folio and the same unbounded extended $(\delta-1)$-folio. Let $G^{\prime}$ be the graph obtained by replacing $G_{1}$ with $G_{1}^{\prime}$ in the separation $\left(G_{1}, G_{2}\right)$. Then $G$ and $G^{\prime}$ have the same $w$-bounded extended $\delta$-folio.

PROOF. The proof is the same as the proof Lemma 2.4 with one additional argument. Suppose first that $\left|E\left(H^{*}\right)\right|+$ is $\left(H^{*}\right) \leq \delta-1$. In this case, we know that $H^{*}$ is in the $(\delta-1)$-folio of $G_{1}^{\prime}+X^{*}$ as well, thus the model $\psi^{\prime}$ exists and the model $\phi^{\prime}$ can be constructed. Note that $R(G)=R\left(G_{2}\right)$, which means that $\phi^{\prime}(v)=\phi(v)$ for every root vertex of $H$ and therefore $\phi^{\prime}$ is $w$-bounded if $\phi$ is $w$-bounded.

Suppose now that $\left|E\left(H^{*}\right)\right|+$ is $\left(H^{*}\right)=\delta$. We claim that in this case $\psi$ is $w$-bounded and hence $H^{*}$ is in the $w$-bounded $\delta$-folio of $G_{1}+X^{*}$ (not only in the unbounded $\delta$-folio). The vertices in $V\left(H^{*}\right) \backslash V(H)$ have degree at most 1 , thus the degree bound holds for such vertices (recall that $w(\psi(v))$ is strictly positive). If a vertex $v \in R\left(H^{*}\right) \cap V(H)$ is in $R(H)$, then $\psi(v)=\phi(v)$ and hence the degree condition holds. Thus we have potential problems only with vertices in $\left(R\left(H^{*}\right) \cap V(H)\right) \backslash R(H)$, i.e., vertices that were already present as non-root vertices in $H$, but became roots in $H^{*}$. The only way such a vertex $u$ could have become a root is if $u$ was incident to a type 3 edge $u v$. If $u$ is isolated in $H^{*}$, then the degree bound immediately holds. If $u$ is not isolated, then the type 3 edge $u v$ does not create any edge or any new isolated vertex in $H^{*}$, thus there is at least one edge of $H$ that does not contribute towards $\left|E\left(H^{*}\right)\right|+$ is $\left(H^{*}\right)$, contradicting $\left|E\left(H^{*}\right)\right|+$ is $\left(H^{*}\right)=\delta$. Thus no such vertex $u$ is possible, and it follows that $\psi$ is $w$-bounded. As $G_{1}$ and $G_{1}^{\prime}$ have the same $w$-bounded extended $\delta$-folio, the model $\psi^{\prime}$ exists, and the rest of the proof is the same as before.

Proposition 2.7. Let $\left(G_{1}, G_{2}\right)$ be a separation of a rooted graph $G$, let $S=V\left(G_{1}\right) \cap V\left(G_{2}\right)$, and suppose that $S \subseteq R(G)$. Let $w$ be a weight function that assigns a positive integer to each vertex of $R(G)$. The w-bounded extended $\delta$-folio of $G$ can be computed from the $w$-bounded extended $\delta$-folio of $G_{1}$, the unbounded extended $(\delta-1)$-folio of $G_{1}$, and the unbounded extended $\delta$-folio of $G_{2}$. 


\section{Algorithmic framework}

The main result of the paper is an algorithm FindFolio that determines the extended $\delta$-folio of the given graph.

FINDFOLIO

Input: $\quad$ Rooted graph $G$, integer $\delta$.

Output: $\quad$ The extended $\delta$-folio of $G$.

THEOREM 3.1. There is an algorithm satisfying the specification of FINDFOLIO that runs in $f_{1}(\delta,|R(G)|) \cdot|V(G)|^{3}$ steps, for some computable function $f_{1}$.

For technical reasons, we prove Theorem 3.1 in the following form:

LEMMA 3.2. There is an algorithm satisfying the specification of FINDFOLIO on instances with $|R(G)| \leq 16 \delta^{2}$ that runs in $f_{1}^{\prime}(\delta)$. $|V(G)|^{3}$ steps, for some computable function $f_{1}^{\prime}$.

It is clear that Lemma 3.2 implies Theorem 3.1: by increasing $\delta$ to, say, $|R(G)|$, the algorithm of Lemma 3.2 can be used even if $|R(G)|$ is arbitrary.

First we design three auxiliary algorithms that either return the extended $\delta$-folio, or some information that is helps our progress: an irrelevant vertex, a clique minor, or an appropriate separation. We say that a set $X$ of vertices is irrelevant to the (extended) $\delta$-folio of $G$, if rooted graphs $G$ and $G \backslash X$ have the same (extended) $\delta$-folio. We say that a vertex $v$ is irrelevant if the set $\{v\}$ is irrelevant. Note that even if every vertex of a set $X$ is irrelevant, the set $X$ need not be irrelevant.

FINDIRRELEVANTORSEPARATION

Input: $\quad$ Rooted graph $G$, integer $\delta$, integer $L$.

Output: $\quad$ - The extended $\delta$-folio of $G$, or

- a vertex $v \in V(G)$ irrelevant to the extended

$\delta$-folio of $G$, or

- a separation $\left(G_{1}, G_{2}\right)$ of $G$ with

$\left|V\left(G_{1}\right)\right|,\left|V\left(G_{2}\right)\right| \geq L$ and having order at most $4 \delta^{2}$.

We say that $B_{1}, \ldots, B_{k}$ are the branch sets of a $K_{k}$-minor, if they are pairwise disjoint, induce connected subgraphs, and for every $1 \leq i<j \leq k$, there is an edge with one endpoint in $B_{i}$ and one endpoint in $B_{j}$.

FINDIRRELEVANTORCLIQUE

Input: $\quad$ Rooted graph $G$, integer $\delta$, integer $k$.

Output: $\quad$ - The $\delta$-folio of $G$, or

- a vertex $v \in V(G)$ irrelevant to the $\delta$-folio of $G$, or

- the branch sets $B_{1}, \ldots, B_{k}$ of a $K_{k}$-minor in $G$.

FINDIRRELEVANTORCLIQUEX

Input: $\quad$ Rooted graph $G$, integer $\delta$, integer $k$.

Output: $\quad$ - The extended $\delta$-folio of $G$, or

- a vertex $v \in V(G)$ irrelevant to the extended $\delta$ -

folio of $G$, or

- the branch sets $B_{1}, \ldots, B_{k}$ of a $K_{k}$-minor in $G$.

THEOREM 3.3. For some computable function $f_{2}$, there is an algorithm satisfying the specification of FINDIRRELEVANTORCLIQUE that runs in $f_{2}(\delta,|R(G)|, k) \cdot|V(G)|$ steps.

Theorem 3.3 is proved in the full version of the paper. It is easy to show that an algorithm for FINDIRRELEVANTORCLIQUE can be used to obtain an algorithm for FINDIRRELEVANTORCLIQUEX:

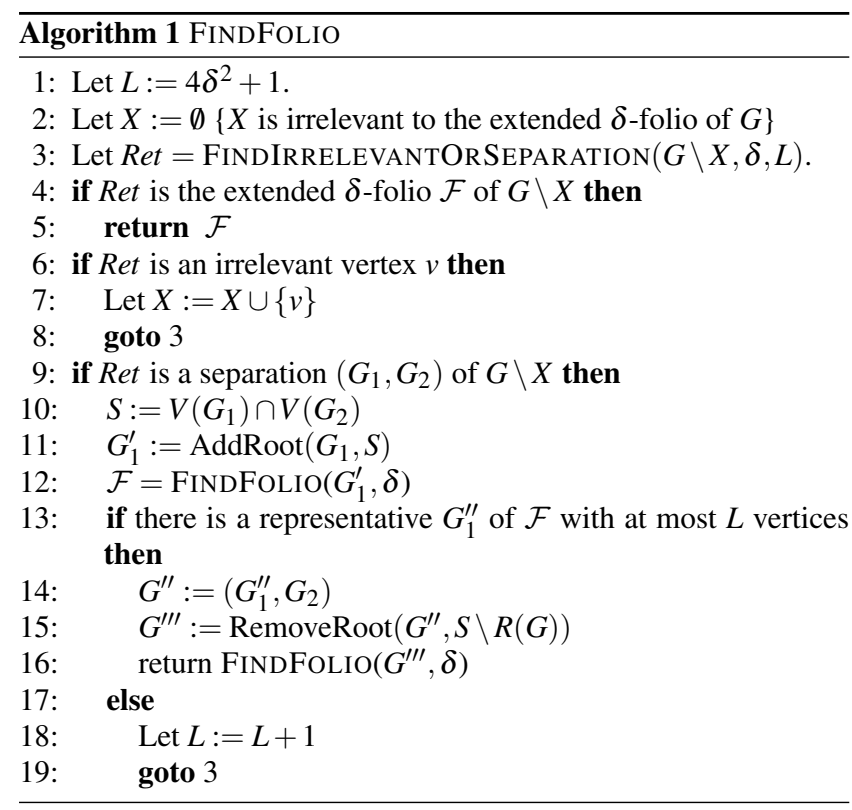

COROLLARY 3.4. For some computable function $f_{2}^{\prime}$, there is an algorithm satisfying the specification of FINDIRRELEVANTORCliQueX that runs in $f_{2}^{\prime}(\delta,|R(G)|, k) \cdot|V(G)|$ steps.

Section 4 presents an algorithm for FINDIRRELEVANTORSEPARATION:

THEOREM 3.5. For some computable function $f_{3}$, there is an algorithm satisfying the specification of FINDIRRELEVANTORSEPARATION that runs in $f_{3}(\delta,|R(G)|, L) \cdot|V(G)|^{2}$ steps.

We prove Theorem 3.5 and Lemma 3.2 by simultaneous induction. In the rest of this section, we prove Lemma 3.2 for some $\delta$, assuming that Theorem 3.5 is true for this $\delta$; while in Section 4, we prove Theorem 3.5 for some $\delta$, assuming that Lemma 3.2 is true for $\delta-1$. It is clear that these two proofs together prove Theorem 3.5 and Lemma 3.2 for every $\delta \geq 0$.

Proof (of Lemma 3.2). Let $L^{*}=\max \left\{L_{\delta, 12 \delta^{2}}, 16 \delta^{2}\right\}$. This constant will be required only for the analysis of the algorithm and it does not appear explictly in the description of the algorithm. Algorithm 1 shows the algorithm in pseudocode. The functions $\operatorname{AddRoot}(G, S)$ and $\operatorname{Remove} \operatorname{Root}(G, S)$ return a rooted graph where $S$ is added to/removed from the set of roots, respectively.

Let $L:=4 \delta^{2}+1$. We will increase $L$ during the algorithm, but (as we shall see) $L \leq L^{*}$ will always hold. Initially we set $X:=\emptyset$; it will always hold that the set of vertices $X$ is irrelevant to the extended $\delta$-folio of $G$.

Let us run algorithm FINDIRRELEVANTORSEPARATION of Theorem 3.5 with $G \backslash X, \delta$, and $L$. If the output is the extended $\delta$ folio of $G \backslash X$, then we are done. If the output is a vertex $v$ irrelevant to the extended $\delta$-folio of $G \backslash X$, then let $X:=X \cup\{v\}$ and call FINDIRRELEVANTORSEPARATION again. It is clear that the new $X$ is irrelevant to the extended $\delta$-folio of $G$. Suppose that (after returning some number of irrelevant vertices) FINDIRRELEVANTORSEPARATION returns a separation $\left(G_{1}, G_{2}\right)$ of $G \backslash X$ with $\left|V\left(G_{1}\right)\right|,\left|V\left(G_{2}\right)\right| \geq L$ and having order at most $4 \delta^{2}$. Note that $L>4 \delta^{2}$, and hence $\left|V\left(G_{1}\right) \backslash V\left(G_{2}\right)\right|,\left|V\left(G_{2}\right) \backslash V\left(G_{1}\right)\right|>0$.

Let $G^{\prime}, G_{1}^{\prime}, G_{2}^{\prime}$ be the same as $G \backslash X, G_{1}$, and $G_{2}$, respectively, with the difference that every vertex of $S=V\left(G_{1}\right) \cap V\left(G_{2}\right)$ is a root (in addition to the original roots). Without loss of generality, we can assume that $\left|R\left(G_{1}\right)\right| \leq\left|R\left(G_{2}\right)\right|$ and hence $\left|R\left(G_{1}^{\prime}\right)\right| \leq$ 
$|R(G)| / 2+|S| \leq 12 \delta^{2}$. Let us call FINDFOLIO recursively to find the extended $\delta$-folio of $G_{1}^{\prime}$ and then let us try to construct by brute force a representative $G_{1}^{\prime \prime}$ of this folio having at most $L$ vertices. If we do not find such a representative, then we increase $L$ by one, and go back to calling FINDIRRELEVANTORSEPARATION (note that this is possible only if $L<L_{\delta, 12 \delta^{2}} \leq L^{*}$, thus we never increase $L$ above $L^{*}$ ). Otherwise, we replace $G_{1}^{\prime}$ with $G_{1}^{\prime \prime}$ in the separation $\left(G_{1}^{\prime}, G_{2}^{\prime}\right)$; let $G^{\prime \prime}$ be the new graph. By Lemma $2.4, G^{\prime}$ and $G^{\prime \prime}$ have the same extended $\delta$-folio. Let $G^{\prime \prime \prime}$ be the graph obtained from $G^{\prime \prime}$ by making those vertices of $S$ non-roots that are non-roots in $G$ (i.e., $\left.\left|R\left(G^{\prime \prime \prime}\right)\right|=|R(G)|\right)$. It is clear that the extended $\delta$-folio of $G \backslash X$ and $G^{\prime \prime \prime}$ are the same. Thus we can finish the algorithm by recursively calling FindFoLIO on $G^{\prime \prime \prime}$ (note that $\left|R\left(G^{\prime \prime \prime}\right)\right| \leq 16 \delta^{2}$ ).

It is obvious from the description that the answer returned by the algorithm is correct. Note that $\left|V\left(G_{1}^{\prime}\right)\right|,\left|V\left(G^{\prime \prime}\right)\right|<|V(G)|$, thus this recursive procedure always terminates.

We need to show that the number of steps can be bounded by $g(\delta) \cdot|V(G)|^{3}$ for some function $g$. The running time required for instances with at most $L^{*}+1$ vertices can be bounded by a constant depending only on $\delta$. We show that there is a function $g^{\prime}$ such that the running time can be bounded by $g^{\prime}(\delta)\left(\mid V(G)-L^{*}-1\right)|V(G)|^{2}$ for instances with $|V(G)|>L^{*}+1$. We prove by induction on $|V(G)|$ that this holds if $g^{\prime}(\delta)$ is sufficiently large.

Let us bound first the number of steps without the calls to FINDIRRELEVANTORSEPARATION and the recursive calls to FINDFOLIO. Let $x$ be the number of times FINDIRRELEVANTORSEPARATION returned an irrelevant vertex. Then FINDIRRELEVANTORSEPARATION was called at most $x+L^{*}$ times (each call either returned an irrelevant vertex or increased $L$, but $L \leq L^{*}$ always hold). Therefore, each line is executed at most $x+L^{*}$ times. Each step can be done in linear time in the size of the graph, thus we can bound the running time by $c_{1} \cdot(x+1)|V(G)|^{2}$ for some constant $c_{1}$ depending on $\delta$. By Theorem 3.5, each call to FIndIRRELEVANTORSEPARATION can be bounded by $f_{3}\left(\delta, 16 \delta^{2}, L\right)|V(G)|^{2}$ steps and the maximum possible value of $L$ is a function of $\delta$, thus the total time required for these calls can be bounded by $c_{2} \cdot(x+$ 1) $|V(G)|^{2}$ for some constant $c_{2}$ depending only on $\delta$.

Finally, let us bound the running time of the recursive calls to FindFolio. If $\left|V\left(G_{1}^{\prime}\right)\right| \leq L^{*}+1$ or $\left|V\left(G^{\prime \prime \prime}\right)\right| \leq L^{*}+1$, then the number of steps of these calls can be bounded by a constant depending only on $\delta$. Let us assume that $\left|V\left(G_{1}^{\prime}\right)\right|,\left|V\left(G^{\prime \prime \prime}\right)\right|>L^{*}+1$. As we noted earlier, $\left|V\left(G_{1}^{\prime}\right)\right|,\left|V\left(G^{\prime \prime \prime}\right)\right|<|V(G)|$, thus the induction hypothesis can be used to bound the running time of these calls. Therefore, the total running time can be bounded as follows:

$$
\begin{gathered}
\left(c_{1}+c_{2}\right)(x+1)|V(G)|^{2}+g^{\prime}(\delta)\left(\left|V\left(G_{1}^{\prime}\right)\right|-L^{*}-1\right)\left|V\left(G_{1}^{\prime}\right)\right|^{2} \\
+g^{\prime}(\delta)\left(\left|V\left(G^{\prime \prime \prime}\right)\right|-L^{*}-1\right)\left|V\left(G^{\prime \prime \prime}\right)\right|^{2} \\
\leq g^{\prime}(\delta)\left((x+1)+\left|V\left(G_{1}^{\prime}\right)\right|-L^{*}-1+\left|V\left(G^{\prime \prime \prime}\right)\right|-L^{*}-1\right)|V(G)|^{2} \\
\leq g^{\prime}(\delta)\left((x+1)+\left|V\left(G_{1}^{\prime}\right)\right|-L^{*}-1+\left|V\left(G_{2}^{\prime}\right) \backslash V\left(G_{1}^{\prime}\right)\right|-1\right)|V(G)|^{2} \\
\leq g^{\prime}(\delta)\left(|V(G)|-L^{*}-1\right)|V(G)|^{2} .
\end{gathered}
$$

In the first inequality, we assume that $g^{\prime}(\delta) \geq c_{1}+c_{2}$. The second inequality follows from $\left|V\left(G^{\prime \prime \prime}\right)\right|=\left|V\left(G_{1}^{\prime \prime}\right) \cup V\left(G_{2}^{\prime}\right)\right|$ and $\left|V\left(G_{1}^{\prime \prime}\right)\right| \leq$ $L \leq L^{*}$. The last inequality follows from $|X|+\left|V\left(G_{1}^{\prime}\right) \cup V\left(G_{2}^{\prime}\right)\right|=$ $|V(G)|$.

\section{Using a large clique minor}

In this section, we prove Theorem 3.5 for some $\delta$, assuming that Lemma 3.2 holds for $\delta-1$. We use the following lemma due to Robertson and Seymour ((5.4) of [11]):
LEMMA 4.1. Let $G$ be a graph and $Z \subseteq V(G)$. Let $k \geq(3 / 2)$. $|Z|$, and let $B_{1}, \ldots, B_{k} \subseteq V(G)$ be the branch sets of a $\bar{K}_{k}$-minor of $G$. Suppose that there is no separation $\left(G_{1}, G_{2}\right)$ of $G$ of order $<|Z|$ with $Z \subseteq V\left(G_{1}\right)$ and $B_{b} \cap V\left(G_{1}\right)=\emptyset$ for some $b \in[k]$. Then for every partition $\left(Z_{1}, \ldots, Z_{n}\right)$ of $Z$ into nonempty subsets there are pairwise disjoint connected subgraphs $T_{1}, \ldots, T_{n} \subseteq G$ such that $V\left(T_{i}\right) \cap Z=Z_{i}$ for all $i \in[n]$.

We say that the $\delta$-folio of a graph is generic if it is as large as possible: it contains every rooted graph $H$ with $|E(H)|+$ is $(H) \leq \delta$ and $\rho_{H}(R(H)) \subseteq \rho_{G}(R(G))$. We say that the $\delta$-folio of a graph is rooted-generic if it contains every such graph $H$ with the additional condition that every vertex of $H$ is rooted (thus generic implies rooted-generic, but not necessarily the other way). The notions of generic and rooted-generic are defined analogously for $w$-bounded folios: in this case we require that every graph that can possibly be present in the $w$-bounded folio is actually present. That is, we require only those graphs $H$ to be in the folio that satisfy the additional condition that for every $v \in R(H)$ and $u \in R(G)$ having the same root numbers, we have $d_{H}(v) \leq w(u)$. We say that the extended ( $w$-bounded) $\delta$-folio is ( $w$-bounded) generic, if this is true for every choice of the set $X$. Note that if $G$ has a generic $\delta$-folio, then $G+X$ has generic $\delta$-folio for any graph $X$ on $R(G)$ : adding edges can only add more graphs to the folio. Thus the extended $\delta$-folio of $G$ is generic if and only if the $\delta$-folio is generic. We can use Lemma 4.1 to obtain sufficient conditions for generic folios:

LEMMA 4.2. Let $G$ be a rooted graph. Let $w$ be a positive integer weight function on $V(G)$. Let $k \geq(3 / 2) \cdot w(R(G))$, and let $B_{1}, \ldots, B_{k} \subseteq V(G)$ be the branch sets of a $K_{k}$-minor of $G$. Suppose that there is no separation $\left(G_{1}, G_{2}\right)$ of $G$ with $w\left(V\left(G_{1}\right) \cap V\left(G_{2}\right)\right)<$ $w(R(G)), R(G) \subseteq V\left(G_{1}\right)$, and $B_{i} \cap V\left(G_{1}\right)=\emptyset$ for some $i \in[k]$.

(1) The $w$-bounded $\delta$-folio of $G$ is rooted-generic.

(2) If there are at least $2 \delta$ vertices $v$ in $R(G)$ with $w(v) \geq 2 \delta$, then the w-bounded $\delta$-folio of $G$ is generic.

PROOF. We need to show that every possible candidate $H$ is in the $w$-bounded $\delta$-folio of $G$. Suppose therefore that $H$ is a rooted graph with $|E(H)|+$ is $(H) \leq \delta, R(H)=V(H)$, and $\rho_{H}(R(H)) \subseteq$ $\rho_{G}(R(G))$. For every $u \in V(H)$, let $\phi(u)$ be the vertex of $G$ with the same root number as $u$ and assume that $d_{H}(u) \leq w(\phi(u))$ for every $u \in V(H)$. We need to show that $H$ is a topological minor of $G$, i.e., $\phi$ can be extended to a model of $H$ in $G$.

For every $v \in V(G)$, let us define $w^{\prime}(v)=d_{H}(u)$ if $v=\phi(u)$ for some $u \in V(H)$, and let $w^{\prime}(v)=w(v)$ if there is no such $u$. Clearly, $w^{\prime}(v) \leq w(v)$ for every $v \in V(G)$ : the degree condition holds for every $v \in R(H)=V(H)$ in $\phi$. Let $G^{\prime}$ be the graph obtained from $G$ by extending each vertex $z \in R(G)$ into a clique $K_{z}$ of size $w^{\prime}(z)$, i.e., we introduce $w^{\prime}(z)-1$ new vertices that are adjacent to each other, to vertex $z$, and to every neighbor of $z$. The clique $K_{z}$ contains $z$ and these $w^{\prime}(z)-1$ new vertices. Let $Z:=\bigcup_{z \in R(G)} K_{z}$. Let us show first that the conditions of Lemma 4.1 hold for $Z$ in $G^{\prime}$. Suppose for contradiction that $\left(G_{1}^{\prime}, G_{2}^{\prime}\right)$ is a separation of $G^{\prime}$ of order less than $|Z|=w^{\prime}(R(G)) \leq w(R(G))$ with $Z \subseteq V\left(G_{1}^{\prime}\right)$ and $B_{b} \subseteq V\left(G_{2}^{\prime}\right) \backslash V\left(G_{1}^{\prime}\right)$ for some $b \in[k]$. Let $S^{\prime}:=$ $V\left(G_{1}^{\prime}\right) \cap V\left(G_{2}^{\prime}\right)$ be the separator. Without loss of generality, we may assume that for all $z \in R(G)$, either $K_{z} \cap S^{\prime}=\emptyset$ or $K_{z} \subseteq S^{\prime}$. Let $G_{1}:=G_{1}^{\prime} \backslash(Z \backslash R(G))$ and $G_{2}:=G_{2}^{\prime} \backslash(Z \backslash R(G))$. Then $\left(G_{1}, G_{2}\right)$ is a separation of $G$; let $S=V\left(G_{1}\right) \cap V\left(G_{2}\right)$ be the separator. Now it is clear that $w(S)=\left|S^{\prime}\right|<|Z|=w^{\prime}(R(G)) \leq w(R(G))$. However, we also have $R(G) \subseteq V\left(G_{1}\right)$ and $B_{b} \cap V\left(G_{1}\right)=\emptyset$, contradicting the assumption of the lemma being proved. Thus we can conclude that there is no such separation $\left(G_{1}^{\prime}, G_{2}^{\prime}\right)$, and the conditions of Lemma 4.1 hold for $Z$ and $G^{\prime}$. 
Let us partition $Z^{\prime}$ in such a way that for every edge $u v \in E(H)$, there is a 2-element class of the partition consisting of a vertex in $K_{\phi(u)}$ and a vertex in $K_{\phi(v)}$. As $K_{\phi(u)}$ and $K_{\phi(v)}$ contain exactly $d_{H}(u)$ and $d_{H}(v)$ vertices, respectively, such a partition exists. Lemma 4.1 gives a set of pairwise disjoint subgraphs, one for each class of the partition. For every edge $u v \in E(H)$, let us denote by $T_{u v}$ the connected subgraph corresponding to the class consisting of a vertex of $K_{\phi(u)}$ and a vertex of $K_{\phi(v)}$, and let us chose a path $P_{u v}^{\prime}$ in $T_{u v}$ that goes from a vertex of $K_{\phi(u)}$ to a vertex of $K_{\phi(v)}$. It is clear that the collection $\mathcal{P}^{\prime}$ of $|E(H)|$ paths obtained this way are pairwise disjoint in $G^{\prime}$. Let us define $P_{u v}$ such that whenever $P_{u v}^{\prime}$ contains a vertex of some $K_{z}$, then we replace it by $z$; let $\mathcal{P}$ be the collection of these paths $P_{u v}$ for every $u v \in E(H)$. Observe that the way $G^{\prime}$ was defined ensures that $P_{u v}$ is a path in $G$. We claim that the paths in $\mathcal{P}$ are pairwise internally disjoint in $G$. As the paths in $\mathcal{P}^{\prime}$ are pairwise disjoint, the only possible problem is that for some $w \in V(H)$, vertex $\phi(w)$ is an internal vertex of some path $P_{u v}$ with $w \notin\{u, v\}$. However, there are $d_{H}(w)=\left|K_{\phi(w)}\right|$ paths in $\mathcal{P}$ whose endpoint is $\phi(w)$ and hence the disjointness $\mathcal{P}^{\prime}$ ensure that there cannot be more than $d_{H}(w)$ paths using vertex $\phi(w)$. We finish the proof of the first statement by extending $\phi$ into a model of $H$ by defining $\phi(u v)$ to be the path $P_{u v}$.

To prove the second statement, let $H$ be a rooted graph with $|E(H)|+$ is $(H) \leq \delta$. Let us obtain $H^{\prime}$ by making every vertex of $H^{\prime}$ a root: if $v \in V(H)$ is not rooted, then let us assign to it a root number that appears on a vertex $v \in R(G)$ with $w(v) \geq \delta$ and is not already used by a vertex of $H$. As $|V(H)| \leq 2 \delta$, the conditions of the lemma show that we can assign root numbers this way. Since the $w$-bounded $\delta$-folio of $G$ is rooted-generic, $H^{\prime}$ is topological minor of $G$, which means that $H$ is also a topological minor of $G$.

We prove Theorem 3.5, under the assumption that Theorem 3.1 is true for $\delta-1$. Let us define the following constants:

$$
\begin{gathered}
h:=2 \delta \\
s:=4 \delta^{2} \\
k:=\max \left\{L, 10 \delta^{2}\right\}+|R(G)|
\end{gathered}
$$

One possible correct output of FINDIRRELEVANTORSEPARATION is a separation $\left(G_{1}, G_{2}\right)$ of $G$ with $\left|V\left(G_{1}\right)\right|,\left|V\left(G_{2}\right)\right| \geq L$ and $\mid V\left(G_{1}\right) \cap$ $V\left(G_{2}\right) \mid \leq s$. We refer to this as finding a small separator.

The algorithm for FINDIRRELEVANTORSEPARATION starts by calling FindirRelevantOrCliQueX for $G, \delta$, and $k$. If FindIRRELEVANTORCLIQUEX returns an irrelevant vertex or the extended $\delta$-folio of $G$, then this is a valid output for FINDIRRELEVANTORSEPARATION as well. Suppose therefore that FINDIRRELEVANTORCLIQUEX returns a $k$-clique minor with branch sets $B_{1}$, $\ldots, B_{k}$. As at most $|R(G)|$ of these sets intersect $R(G)$, we can assume without loss of generality that $B_{1}, \ldots, B_{L}$ are disjoint from $R(G)$.

The rest of the section discusses two cases depending on the number of vertices with degree at least $L$ in $G^{\prime}$.

\subsection{Case 1: Many high-degree vertices}

Suppose that there are at least $h$ vertices with degree at least $L$. Let $U$ be a set of $h$ such vertices.

Let us enumerate every nonempty subset of size at most $2 \delta$ of $|R(G)|$; let $R_{1}, \ldots, R_{t}$ be these subsets. Let $w_{i}$ be a weight assignment on $V(G)$ such that $w(v)=\delta$ if $v \in R_{i} \cup U$ and $w(v)=1$ otherwise. By Proposition 3, the folio of $G$ can be obtained from the folios $G$ with respect to $R_{1}, \ldots, R_{t}$. Furthermore, the $w_{i}$-bounded $\delta$-folio of $G$ with respect to $R_{i}$ is obviously the same as the unbounded $\delta$-folio with respect to $R_{i}$.
For every $1 \leq i \leq t$, we compute a separation $\left(G_{1}^{i}, G_{2}^{i}\right)$ of $G$ such that $R_{i} \cup U \subseteq V\left(G_{1}^{i}\right)$, there is a $1 \leq b \leq L$ with $B_{b} \subseteq V\left(G_{2}^{i}\right) \backslash V\left(G_{1}^{i}\right)$, and $w_{i}\left(V\left(G_{1}^{i}\right) \cap V\left(G_{2}^{i}\right)\right)$ is as small as possible. Such a separation $\left(G_{1}^{i}, G_{2}^{i}\right)$ can be done by running, for every $1 \leq j \leq L$, a weighted minimum vertex cutset algorithm to find a set of vertices that separates $R_{i} \cup U$ and $B_{j}$; among these $L$ separations, we define $\left(G_{1}^{i}, G_{2}^{i}\right)$ to be the one that minimizes $w_{i}\left(V\left(G_{1}^{i}\right) \cap V\left(G_{2}^{i}\right)\right)$. Let $S_{i}:=V\left(G_{1}^{i}\right) \cap$ $V\left(G_{2}^{i}\right)$.

Note that $\left(G\left[R_{i} \cup U\right], G \backslash E\left(G\left[R_{i} \cup U\right]\right)\right)$ is always a separation that satisfies the requirements, thus we can assume that $w_{i}\left(S_{i}\right) \leq$ $w\left(R_{i} \cup U\right) \delta(2 \delta+h)=s$. As each of $B_{1}, \ldots, B_{L}$ intersects $V\left(G_{2}^{i}\right)$, we have $\left|V\left(G_{2}^{i}\right)\right| \geq L$. This means that if $\left|V\left(G_{1}^{i}\right)\right| \geq L$ also holds, then separation $\left(G_{1}^{i}, G_{2}^{i}\right)$ is a small separation that can be returned as a valid output of FindIRRELEVANTORSEPARATION. Thus we can assume in the following that $\left|V\left(G_{1}^{i}\right)\right|<L$. This implies that $U \subseteq S_{i}$ : if some $u \in U$ is not in $S_{i}$, then every neighbor of $u$ is in $V\left(G_{1}^{i}\right)$, and $\left|V\left(G_{1}^{i}\right)\right| \geq L$ follows.

We use Lemma 4.2 to show that the $w_{i}$-bounded $\delta$-folio of $G_{2}^{i}$ is generic with respect to $S_{i}$. At most $\left|S_{i}\right| \leq w_{i}\left(R_{i} \cup U\right) \leq \delta\left(\left|R_{i}\right|+\right.$ $|U|) \leq 4 \delta^{2}$ of the sets $B_{1}, \ldots, B_{L}$ intersect $S_{i}$, thus we can suppose without loss of generality that $B_{1}, \ldots, B_{6 \delta^{2}}$ are disjoint from $S_{i}$. Suppose that $G_{2}^{i}$ has a separation $\left(F_{1}, F_{2}\right)$ contradicting the conditions of Lemma 4.2: $S_{i} \subseteq V\left(F_{1}\right), B_{b} \subseteq V\left(F_{2}\right) \backslash V\left(F_{1}\right)$ for some $1 \leq b \leq 6 \delta^{2}$, and $w_{i}\left(V\left(F_{1}\right) \cap V\left(F_{2}\right)\right)<w_{i}\left(S_{i}\right)$. Such a separation can be extended to a separation $\left(F_{1}^{\prime}, F_{2}^{\prime}\right)$ of $G$ with $V\left(G_{1}^{i}\right) \subseteq V\left(F_{1}^{\prime}\right)$, $V\left(F_{1}^{\prime}\right) \cap V\left(F_{2}\right)=V\left(F_{1}\right) \cap V\left(F_{2}\right)$ and $B_{b} \subseteq V\left(F_{2}^{\prime}\right) \backslash V\left(F_{1}^{\prime}\right)$. However, such a separation would contradict the minimality of the choice of $S_{i}$. Thus the conditions of Lemma 4.2 hold, and the $w_{i}$-bounded $\delta$-folio of $G_{2}^{i}$ is generic with respect to $S_{i}$.

We use Proposition 2.7 to compute the $w_{i}$-bounded $\delta$-folio of $G$ with respect to $R_{i} \cup S_{i}$; by Proposition 2.3(2), this can be used to compute the $w_{i}$-bounded $\delta$-folio of $G$ with respect to $R_{i}$. As $\left|V\left(G_{1}^{i}\right)\right|<L$, the extended $\delta$-folio of $G_{1}^{i}$ with respect to $R_{i} \cup S_{i}$ can be determined by brute force in time depending only on $L$. We can determine the (unbounded) extended $(\delta-1)$-folio of $G_{2}^{i}$ with respect to $S_{i}$ by calling FINDFOLIO (recall that we assume in this section that Lemma 3.2 holds for $\delta-1$ and $\left|S_{i}\right| \leq 4 \delta^{2} \leq 16(\delta-1)^{2}$, satisfying the conditions of Lemma 3.2). We have shown above that the extended $w_{i}$-bounded $\delta$-folio of $G_{2}^{i}$ with respect to $S_{i}$ is generic. Thus we have all the information required by Prop. 2.7 at our disposal to compute the $w_{i}$-bounded $\delta$-folio of $G$ with respect to $R_{i} \cup S_{i}$.

\subsection{Case 2: Few high-degree vertices}

Let $U$ be the set of all vertices in $G^{\prime}$ with degree at least $L$; we suppose in this case that $|U|<h$. To determine the extended $\delta$-folio of $G$, for every graph $X$ on $R(G)$, we need to determine the $\delta$-folio of $G+X$. Fixing such an $X$, we set $G^{\prime}=G+X$ and proceed the following way.

We define a graph $F$ on vertex set $V\left(G^{\prime}\right) \backslash U$, where two vertices are adjacent if their distance in $G^{\prime} \backslash U$ is at most $2 L$. As the maximum degree of $G^{\prime} \backslash U$ is at most $L^{\prime}=L+|R(G)|$, the maximum degree of $F$ is at most $\left(L^{\prime}\right)^{2 L^{\prime}+1}$. We say that a subset $C \subseteq V\left(G^{\prime}\right) \backslash U$ of vertices is a cluster if $F[C]$ is connected. The maximum number of clusters of size at most $x$ that contain a vertex $v \in V\left(G^{\prime}\right) \backslash U$ can be bounded by a function of the maximum degree of $F$ and $x$. Therefore, assuming $\delta,|R(G)|$, and $L$ are fixed constants, the total number of clusters of size at most $2 \delta$ is linear in $\left|V\left(G^{\prime}\right)\right|$. Let $C_{1}$, $\ldots, C_{t}$ be an enumeration of the clusters of size at most $2 \delta$.

For every $1 \leq i \leq t$, let $w_{i}$ be a weight function on $V\left(G^{\prime}\right) \backslash U$ defined as $w_{i}(v)=\delta$ for $v \in C_{i}$ and $w_{i}(v)=1$ otherwise. For ev- 
ery $1 \leq i \leq t$, let us choose a separation $\left(G_{1}^{i}, G_{2}^{i}\right)$ of $G^{\prime} \backslash U$ such that $C_{i} \subseteq V\left(G_{1}^{i}\right)$, there is a branch set $B_{b}$ with $B_{b} \subseteq V\left(G_{2}^{i}\right) \backslash V\left(G_{1}^{i}\right)$, and $w_{i}\left(V\left(G_{1}^{i}\right) \cap V\left(G_{2}^{i}\right)\right)$ is minimum possible. It is easy to see that we can choose the separation such that every connected component of $G_{1}^{i}$ contains a vertex of $C_{i}$. Let $D_{i}=V\left(G_{1}^{i}\right)$ and $S_{i}=V\left(G_{1}^{i}\right) \cap$ $V\left(G_{2}^{i}\right)$. The separation $\left(G^{\prime}\left[C_{i}\right], G^{\prime} \backslash E\left(G^{\prime}\left[C_{i}\right]\right)\right)$ and the minimality of $w_{i}\left(S_{i}\right)$ shows that $w_{i}\left(S_{i}\right) \leq w_{i}\left(C_{i}\right) \leq 2 \delta \cdot \delta$ and hence $\left|S_{i}\right| \leq$ $w_{i}\left(S_{i}\right) \leq 2 \delta^{2}$. Every branch set of the clique intersects $V\left(G_{2}^{i}\right)$, which means that $\left|V\left(G_{2}^{i}\right)\right| \geq L$. If $\left|V\left(G_{1}^{i}\right)\right| \geq L$ also holds, then $G^{\prime}$ has a small separation $\left(G_{1}, G_{2}\right)$ with $V\left(G_{1}\right)=V\left(G_{1}^{i}\right) \cup u, V\left(G_{2}\right)=$ $V\left(G_{2}^{i}\right) \cup U$, and $\left|V\left(G_{1}\right) \cap V\left(G_{2}\right)\right|=\left|V\left(G_{1}^{i}\right) \cap V\left(G_{2}^{i}\right)\right|+|U| \leq s$, which we can return. Thus in the following, we can assume that $\left|D_{i}\right|<L$.

We say that two clusters $C_{i_{1}}$ and $C_{i_{2}}$ are independent if there is no edge between $C_{i_{1}}$ and $C_{i_{2}}$ in $F$.

PROPOSITION 4.3. If clusters $C_{i_{1}}$ and $C_{i_{2}}$ are independent, then $D_{i_{1}} \cap D_{i_{2}}=\emptyset$.

PROOF. Let us choose a vertex $v \in D_{i_{1}} \cap D_{i_{2}}$. As $\left|D_{i_{1}}\right|<L$ and the component of $G_{1}^{i_{1}}$ containing $v$ contains a vertex of $C_{i_{1}}$, vertex $v$ is at distance at most $L$ from some vertex of $C_{i_{1}}$ in $G_{1}^{i_{1}}$, and therefore in $G^{\prime} \backslash U$. Similarly, $v$ is at distance at most $L$ from some vertex of $C_{i_{2}}$ in $G^{\prime} \backslash U$. Thus there is an edge in $F$ between a vertex of $C_{i_{1}}$ and a vertex of $C_{i_{2}}$, a contradiction.

DEFINITION 4.4. We say that clusters $C_{i_{1}}$ and $C_{i_{2}}$ are equivalent if there is a rooted isomorphism between the graphs $G\left[D_{i_{1}} \cup U\right]$ and $G\left[D_{i_{2}} \cup U\right]$ that is the identity on $U$, maps $S_{i_{1}}$ to $S_{i_{2}}$, and maps $C_{i_{1}}$ to $C_{i_{2}}$.

The following proposition is easy to prove:

PROPOSITION 4.5. The number of equivalence classes of the clusters can be bounded by a function of $\delta$ and $L$.

As we shall see, the topological minor is realized by a small number of clusters and paths connecting them. The following definition tries to capture which paths are inside a cluster and which paths are between clusters.

DEFINITION 4.6. Let $H$ be a rooted graph. A scheme of $H$ is a pair $\left(H^{\prime}, H_{*}^{\prime}\right)$ of rooted graphs, where

(1) $H^{\prime}$ is a subdivision of $H$ (the new vertices are not roots),

(2) $H_{*}^{\prime}$ is a subgraph of $H^{\prime}$, and

(3) every vertex of $V\left(H_{*}^{\prime}\right) \backslash V(H)$ has degree at most 1 in $H_{*}^{\prime}$.

For every $r$-tuple $\mathcal{C}=\left(C_{i_{1}}, \ldots, C_{i_{r}}\right)$ of clusters, we define $C^{\mathcal{C}}=$ $\bigcup_{i=1}^{r} C_{i_{j}}, D^{\mathcal{C}}=\bigcup_{i=1}^{r} D_{i_{j}}$, and $S^{\mathcal{C}}=\bigcup_{i=1}^{r} S_{i_{j}}$. We define two graphs: $G_{1}^{\mathcal{C}}=G^{\prime}\left[U \cup D^{\mathcal{C}}\right]$ and $G_{2}^{\mathcal{C}}=G^{\prime} \backslash\left(D^{\mathcal{C}} \backslash S^{\mathcal{C}}\right)$. Note that $\left(G_{1}^{\mathcal{C}}, G_{2}^{\mathcal{C}}\right)$ is a separation of $G$. We also define a weight function $w^{\mathcal{C}}$ on $V(G)$ that is $\delta$ on every vertex of $U \cup C^{\mathcal{C}}$ and 1 on every other vertex.

DEFINITION 4.7. Let $H$ be a rooted graph and let $\left(H^{\prime}, H_{*}^{\prime}\right)$ be a scheme of $H$. Let $\mathcal{C}=\left(C_{i_{1}}, \ldots, C_{i_{r}}\right)$ be an r-tuple of clusters. We say that this tuple realizes the scheme $\left(H^{\prime}, H_{*}^{\prime}\right)$ if $H^{\prime} \backslash E\left(H_{*}^{\prime}\right)$ has a model $\phi$ in $G_{1}^{\mathcal{C}}$ such that

(1) every vertex of $V(H)$ is mapped to $U \cup C^{\mathcal{C}}$,

(2) every vertex of $V\left(H_{*}^{\prime}\right)$ is mapped to $U \cup S^{\mathcal{C}}$, and

(3) for every $e \in E\left(H^{\prime}\right) \backslash E\left(H_{*}^{\prime}\right)$, the internal vertices of $\phi(e)$ are not in $U \cup S^{\mathcal{C}}$.
Roughly speaking, what we want to show is that $H$ is a topological minor of $G$ if and only if there is a tuple of independent clusters that realizes a scheme of $H$ (Lemmas 4.8 and 4.10). Therefore, deciding whether $H$ is a topological minor essentially reduces to finding a tuple of independent clusters that realize a given scheme of $H$. As the clusters can be classified into a bounded number of equivalence classes, the main difficulty is to find independent clusters of given types, which can be solved using standard techniques.

We first prove that if a rooted graph $H$ has a model in $G^{\prime}$, then $H$ has a scheme that is realized by some tuple of clusters. We hope the proof sheds light on why schemes are defined this way.

LEMMA 4.8. Let $H$ be a rooted graph in the $\delta$-folio of $G^{\prime}$. Then there is a scheme $\left(H^{\prime}, H_{*}^{\prime}\right)$ of $H$ with $\left|V\left(H^{\prime}\right)\right| \leq 4 \delta+2 \delta^{2}$ and a tuple $\mathcal{C}=\left(C_{i_{1}}, \ldots, C_{i_{r}}\right)$ of pairwise independent clusters with $r \leq$ $2 \delta$ that realizes $\left(H^{\prime}, H_{*}^{\prime}\right)$.

Proof. Let $\phi$ be a model of $H$ in $G^{\prime}$. Let $C=\{\phi(v) \mid v \in$ $V(H)\} \backslash U$. Each connected component of $F[C]$ is a cluster; let $\mathcal{C}=\left(C_{i_{1}}, \ldots, C_{i_{r}}\right)$ be these connected components. Clearly, these clusters are pairwise independent and $r \leq|V(H)| \leq 2 \delta$. Due to a minor technical detail, we need to handle some vertices of $S^{\mathcal{C}} \cup U$ in a special way. We define $X$ to contain a vertex $v \in S^{\mathcal{C}} \cup U$ if $v$ is an internal vertex of $\phi(e)$ for some $e \in E(H)$ and both neighbors of $v$ in $\phi(e)$ are in $V\left(G_{2}^{\mathcal{C}}\right)$.

If for some $e \in V(H)$, the path $\phi(e)$ contains $m$ internal vertices in $\left(S^{\mathcal{C}} \cup U\right) \backslash X$, then let us subdivide $e$ with $m$ new (nonroot) vertices; let $H^{\prime}$ be the rooted graph obtained this way. As $\left|S^{\mathcal{C}} \cup U\right| \leq 2 \delta^{2}+2 \delta$, we have $\left|V\left(H^{\prime}\right)\right| \leq 4 \delta+2 \delta^{2}$. The model $\phi$ gives a model $\phi^{\prime}$ of $H$ in $G$ the obvious way (every new vertex of the subdivision is mapped to a vertex in $\left.\left(S^{\mathcal{C}} \cup U\right) \backslash X\right)$. Let $H_{*}^{\prime}$ be the subgraph of $H^{\prime}$ that contains those vertices $v$ for which $\phi^{\prime}(v) \in\left(S^{\mathcal{C}} \cup U\right) \backslash X$ and those edges $e$ for which $\phi^{\prime}(e)$ is fully contained in $G_{2}^{\mathcal{C}}$.

We claim that $\left(H^{\prime}, H_{*}^{\prime}\right)$ is a scheme of $H$ and $\mathcal{C}$ realizes this scheme. Conditions 1 and 2 of Definition 4.6 are easy to verify. To check condition 3 , suppose that vertex $v \in V\left(H_{*}^{\prime}\right) \backslash V(H)$ has degree more than 1 . Since vertex $v$ was obtained as the subdivision of an edge $e \in E(H)$, vertex $v$ has degree exactly 2 in $H_{*}^{\prime}$ and $\phi^{\prime}(v) \in\left(S^{\mathcal{C}} \cup U\right) \backslash X$. Let $e_{1}$ and $e_{2}$ be the two edges incident to $v$ in $H_{*}^{\prime}$. By definition of $H_{*}^{\prime}, \phi^{\prime}\left(e_{1}\right)$ and $\phi^{\prime}\left(e_{2}\right)$ are fully contained in $G_{2}^{\mathcal{C}}$. Thus the two neighbors of $\phi(v)$ in $\phi(e)$ are both in $V\left(G_{2}^{\mathcal{C}}\right)$, implying that $\phi(v) \in X$, a contradiction.

Finally, we show that $\phi^{\prime}$ defines a model of $H^{\prime} \backslash E\left(H_{*}^{\prime}\right)$ in $G_{1}^{\mathcal{C}}$ satisfying the conditions of Definition 4.7. Let us verify that the images of the vertices and edges are indeed in $G_{1}^{\mathcal{C}}$. It is clear that $\phi^{\prime}(v) \in V\left(G_{1}^{\mathcal{C}}\right)$ for every $v \in V\left(H^{\prime}\right)$. Let us prove that $\phi^{\prime}(e)$ is fully contained in $V\left(G_{1}^{\mathcal{C}}\right)$ for every $e \in E\left(H^{\prime}\right) \backslash E\left(H_{*}^{\prime}\right)$. In fact, we show that $\phi^{\prime}(e)$ has no internal vertex in $V\left(G_{2}^{\mathcal{C}}\right)$. Suppose that $\phi^{\prime}(e)$ has an internal vertex $u_{2} \in V\left(G_{2}^{\mathcal{C}}\right)$. As $e \notin E\left(H_{*}^{\prime}\right)$, path $\phi^{\prime}(e)$ contains a vertex $u_{1} \in V\left(G_{1}^{\mathcal{C}}\right) \backslash V\left(G_{2}^{\mathcal{C}}\right)\left(u_{1}\right.$ can be an endpoint of $\left.\phi^{\prime}(e)\right)$. Going from $u_{1}$ to $u_{2}$ on $\phi^{\prime}(e)$, let $u$ be the first vertex of $V\left(G_{2}^{\mathcal{C}}\right)$; clearly, $u \in S \cup U$ and $u \neq u_{1}$. Now $u$ is an internal vertex of $\phi^{\prime}(e)$, and the vertex preceding $u$ is not in $V\left(G_{2}^{\mathcal{C}}\right)$. Thus $u \in\left(S^{\mathcal{C}} \cup U\right) \backslash X$, which means that $u$ should be the image of a vertex of $H^{\prime}$ in $\phi^{\prime}$, a contradiction. Therefore, $\phi^{\prime}(e)$ has no internal vertex in $V\left(G_{2}^{\mathcal{C}}\right)$ and in particular $\phi^{\prime}(e)$ is fully contained in $V\left(G_{1}^{\mathcal{C}}\right)$ for every $e \in$ $E\left(H^{\prime}\right) \backslash E\left(H_{*}^{\prime}\right)$. This means that $\phi^{\prime}$ is indeed a model of $H^{\prime} \backslash E\left(H_{*}^{\prime}\right)$ in $G_{1}^{\mathcal{C}}$ and we also verified condition 3 of Definition 4.7. Conditions 1 and 2 are straightforward to check.

We prove now the converse of Lemma 4.8. We show first that the $w_{i}$-bounded folio of $G_{2}^{\mathcal{C}}$ is rooted-generic (Lemma 4.9). Then 
we use this fact to route the edges of $H_{*}^{\prime}$ when constructing a model of $H^{\prime}$ in $G^{\prime}$ (Lemma 4.10).

LEMMA 4.9. Let $\mathcal{C}=\left(C_{i_{1}}, \ldots, C_{i_{r}}\right)$ be a tuple of pairwise independent clusters. Either the $w^{\mathcal{C}}$-bounded $w^{\mathcal{C}}\left(S^{\mathcal{C}}\right)$-folio of $G_{2}^{\mathcal{C}}$ with respect to $U \cup S^{\mathcal{C}}$ is rooted-generic (and we can find a model of every graph in the folio), or we can find a separation $\left(G_{1}^{\prime}, G_{2}^{\prime}\right)$ of $G^{\prime}$ with $\left|V\left(G_{1}^{\prime}\right)\right|,\left|V\left(G_{2}^{\prime}\right)\right| \geq L$ and $\left|V\left(G_{1}^{\prime}\right) \cap V\left(G_{2}^{\prime}\right)\right| \leq s$.

PROOF. If the conditions of Lemma 4.2 hold for $G_{2}^{\mathcal{C}}, w^{\mathcal{C}}$, and set of roots $U \cup S^{\mathcal{C}}$, then we are done. Suppose therefore that there is a separation $\left(F_{1}, F_{2}\right)$ of $G_{2}^{\mathcal{C}}$ violating the conditions of Lemma 4.2. There is a corresponding separation $\left(G_{1}^{\prime}, G_{2}^{\prime}\right)$ of $G^{\prime}$ with $V\left(F_{1}\right) \cap$ $V\left(F_{2}\right)=V\left(G_{1}^{\prime}\right) \cap V\left(G_{2}^{\prime}\right), V\left(G_{1}^{\prime}\right) \subseteq V\left(F_{1}\right)$, and $V\left(G_{2}^{\prime}\right)=V\left(F_{2}\right)$. Let $S^{\prime}=V\left(F_{1}\right) \cap V\left(F_{2}\right)=V\left(G_{1}^{\prime}\right) \cap V\left(G_{2}^{\prime}\right)$, it is clear that $\left|S^{\prime}\right| \leq$ ${ }^{\mathcal{C}}\left(U \cup S^{\mathcal{C}}\right) \leq s$. As $B_{b} \subseteq V\left(G_{2}^{\prime}\right)$, we also have $\left|V\left(G_{2}^{\prime}\right)\right| \geq L$. If $\left|V\left(G_{1}^{\prime}\right)\right| \geq L$, then we can return the small separation $\left(G_{1}^{\prime}, G_{2}^{\prime}\right)$. Thus in the following, we can assume that $\left|V\left(G_{1}^{\prime}\right)\right| \leq L$. In particular, this means that $U \subseteq S^{\prime}$ : if $u \in V\left(G_{1}^{\prime}\right) \backslash V\left(G_{2}^{\prime}\right)$ for some $u \in U$, then every neighbor of $u$ is in $V\left(G_{1}^{\prime}\right)$ and $\left|V\left(G_{1}^{\prime}\right)\right| \geq L$ follows.

Let $S_{i_{j}}^{\prime}$ be the set of those vertices of $S^{\prime} \backslash U$ that can be reached from $S_{i_{j}} \subseteq V\left(G_{1}^{\prime}\right) \backslash U$ by a path in $G_{1}^{\prime} \backslash U$. We claim that these sets are pairwise disjoint for $j=1, \ldots, r$. Suppose without loss of generality that there is a vertex $v \in S_{i_{1}}^{\prime} \cap S_{i_{2}}^{\prime}$. This means that there is a vertex $v_{1} \in S_{i_{1}}$ and a vertex $v_{2} \in S_{i_{2}}$ that are in the same connected component $K$ of $G_{1}^{\prime} \backslash U$ as $v$. Note that $D_{i_{1}}$ and $D_{i_{2}}$ are fully contained in $G_{1}^{\prime} \backslash U$, thus there is a vertex $c_{1} \in C_{i_{1}}$ and a vertex $c_{2} \in C_{i_{2}}$ in this connected component $K$. As clusters $C_{i_{1}}$ and $C_{i_{2}}$ are independent by assumption, the distance of $c_{1}$ and $c_{2}$ is at least $2 L$ in $G_{1}^{\prime} \backslash U$, which means that $\left|V\left(G_{1}^{\prime}\right)\right| \geq 2 L$, a contradiction.

As $U, S_{i_{1}}^{\prime}, \ldots, S_{i_{r}}^{\prime}$ are pairwise disjoint and $U \subseteq S$, the only way ${ }^{\mathcal{C}}\left(S^{\prime}\right)<w^{\mathcal{C}}(S)$ is possible if $w^{\mathcal{C}}\left(S_{i_{j}}^{\prime}\right)<w^{\mathcal{C}}\left(S_{i_{j}}\right)$ for some $1 \leq j \leq$ $r$. However, in this case there is a separation $\left(G_{1}^{i_{j}}, G_{2}^{i_{j}}\right)$ of $G^{\prime} \backslash$ $U$ with $V\left(G_{1}^{i_{j}}\right) \cap V\left(G_{2}^{i_{j}}\right)=S_{i_{j}}^{\prime}, D_{i_{j}} \subseteq V\left(G_{1}^{i_{j}}\right)$, and $B_{b} \subseteq V\left(G_{2}^{i_{j}}\right) \backslash$ $V\left(G_{1}^{i_{j}}\right)$ for some branch set $B_{b}$. This contradicts the minimality of the choice of $S_{i_{j}}$.

LEMMA 4.10. Let $H$ be a rooted graph and $\left(H^{\prime}, H_{*}^{\prime}\right)$ be a scheme of $H$. Let $\mathcal{C}=\left(C_{i_{1}}, \ldots, C_{i_{r}}\right)$ be an r-tuple of pairwise independent clusters that realizes $\left(H^{\prime}, H_{*}^{\prime}\right)$. Then we can find either a model of $H$ in $G^{\prime}$ or a separation $\left(G_{1}^{\prime}, G_{2}^{\prime}\right)$ of $G^{\prime}$ with $\left|V\left(G_{1}^{\prime}\right)\right|,\left|V\left(G_{2}^{\prime}\right)\right| \geq L$ and $\left|V\left(G_{1}^{\prime}\right) \cap V\left(G_{2}^{\prime}\right)\right| \leq s$.

Proof. Let $\phi$ be a model of $H^{\prime} \backslash E\left(H_{*}^{\prime}\right)$ in $G_{1}^{\mathcal{C}}$, as in Definition 4.7. Since $G_{1}^{\mathcal{C}}$ is a subgraph of $G^{\prime}, \phi$ can be considered as a model of $H^{\prime} \backslash E\left(H_{*}^{\prime}\right)$ in $G^{\prime}$. We try to extend $\phi$ to a model of $H^{\prime}$ in $G^{\prime}$ by assigning values to $\phi(e)$ for every $e \in E\left(H_{*}^{\prime}\right)$. In order to do this, let us make every vertex of $U \cup S^{\mathcal{C}}$ a root $G_{2}^{\mathcal{C}}$, and let $H_{*}^{\prime \prime}$ be obtained from $H_{*}^{\prime}$ by making every vertex $v$ a root with the same root number as $\psi(v)$. We try to find a $w^{\mathcal{C}}$-bounded model $\psi$ of $H_{*}^{\prime}$ in $G_{2}^{\mathcal{C}}$. Note that Definition 4.7 ensures that such a $\psi$ respects the degree condition: for every $v \in V\left(H_{*}^{\prime}\right) \cap V(H)$, we have $\psi(v) \in U \cup S^{\mathcal{C}}$ and hence $w^{C}(\psi(v))=\delta$, while the degree of every $v \in V\left(H_{*}^{\prime}\right) \backslash V(H)$ is at most 1 in $H_{*}^{\prime}$. We use Lemma 4.9 to find either a small separation $\left(G_{1}^{\prime}, G_{2}^{\prime}\right)$, or a model $\psi$ of $H_{*}^{\prime \prime}$ in $G_{2}^{\mathcal{C}}$ with $\psi(v)=\phi(v)$ for every $v \in V(H)$. If Lemma 4.9 gives us a separation, then we are done. Otherwise, let us set $\phi(e)=\psi(e)$ for every $e \in E\left(H_{*}^{\prime}\right)$. The paths $\phi(e)$ for $e \in E\left(H_{*}^{\prime}\right)$ are pairwise internally disjoint: this follows from the fact that if $e \in E\left(H_{*}^{\prime}\right)$, then the internal vertices of $\phi(e)=\psi(e)$ are in $V\left(G_{2}^{\mathcal{C}}\right)$, while for every $e \in E\left(H^{\prime}\right) \backslash E\left(H_{*}^{\prime}\right)$, the internal vertices of $\phi(e)$ are not in $V\left(G_{2}^{\mathcal{C}}\right)$ (by Definition 4.7(3)). Thus $\phi$ is indeed a model of $H^{\prime}$.
Having established the correspondence between topological minors and tuples of clusters realizing a scheme, we concentrate on finding such a tuple. We observe that only the equivalence types of the clusters matter:

PROPOSITION 4.11. Let $H$ be a rooted graph and $\left(H^{\prime}, H_{*}^{\prime}\right)$ be a scheme of $H$. Let $\left(C_{i_{1}}, \ldots, C_{i_{r}}\right)$ and $\left(C_{i_{1}^{\prime}}, \ldots, C_{i_{r}^{\prime}}\right)$ be two r-tuple of clusters such that $\left(C_{i_{1}}, \ldots, C_{i_{r}}\right)$ realizes $\left(H^{\prime}, H_{*}^{\prime}\right)$ and for every $1 \leq j \leq r$, clusters $C_{i_{j}}$ and $C_{i_{j}^{\prime}}$ are equivalent. Then $\left(C_{i_{1}^{\prime}}, \ldots, C_{i_{r}^{\prime}}\right)$ also realizes $\left(H^{\prime}, H_{*}^{\prime}\right)$.

The following lemma is standard: it shows that finding small fixed-size "colorful" independent sets in bounded-degree graphs can be done in linear time.

LEMMA 4.12. Let $W$ be a graph with maximum degree $d$ where the vertices are labeled with $k$ different labels. We can find in time $f(d, k) \cdot(|V(W)|+|E(W)|)$ an independent set of size $k$ where every vertex has a different label (or correctly state that there is no such set).

LEMMA 4.13. Given a scheme $\left(H^{\prime}, H_{*}^{\prime}\right)$ with $\left|V\left(H^{\prime}\right)\right| \leq 4 \delta+$ $2 \delta^{2}$, in time $f(\delta, L)|V(G)|$ (for some function $f(\delta)$ ) we can find a tuple $\mathcal{C}=\left(C_{i_{1}}, \ldots, C_{i_{r}}\right)$ of clusters with $r \leq 2 \delta$ that realizes $\left(H^{\prime}, H_{*}^{\prime}\right)$ (if such a tuple exists).

PROOF. Let us enumerate all clusters and sort them into equivalence classes (where equivalence is understood according to Definition 4.4). Let $t$ be the number of equivalence classes and let us assign an integer $\tau\left(C_{i}\right) \in[t]$ to each cluster $C_{i}$ based on which class it belongs to. For every subset $T \subseteq[t]$ of size at most $2 \delta$, we test whether there is a tuple $\left(C_{i_{1}}, \ldots, C_{i_{T \mid}}\right)$ of pairwise independent clusters with $\left\{\tau\left(C_{i_{1}}\right), \ldots, \tau\left(C_{i_{|T|}}\right)\right\}=T$. In order to do this, we build a graph $W_{T}$ by introducing a vertex with label $\tau\left(C_{i}\right)$ corresponding to every cluster $C_{i}$ with $\tau\left(C_{i}\right) \in T$. Two vertices of $W_{T}$ are adjacent if the corresponding clusters are not independent. We claim that the maximum degree of $W_{T}$ can be bounded by a function of $\delta$ and $L$. To see this, recall that the maximum degree of $G \backslash U$ is at most $L$ and that the maximum distance in $G \backslash U$ between two vertices of a cluster $C_{i}$ is $O(\delta L)$ (as $C_{i}$ induces a connected subgraph of $F$ ). Thus if $C_{i}$ and $C_{j}$ are not independent, then $C_{j}$ is fully contained in the $O(\delta L)$-neighborhood of every vertex of $C_{i}$; the number of such sets can be bounded by a function of $\delta$ and $L$. This means that if we use Lemma 4.12 to find a colorful independent set in $W_{T}$, then the running time is linear in the number of clusters (for fixed $\delta$ and $L$ ). If Lemma 4.12 returns an independent set, then we test if the corresponding pairwise independent tuple $\mathcal{C}=\left(C_{i_{1}}, \ldots, C_{i_{|T|}}\right)$ of clusters realizes $\left(H^{\prime}, H_{*}^{\prime}\right.$ ) (as the size of $G_{1}^{\mathcal{C}}$ is bounded by a function of $\delta$ and $L$, this can be done by brute force). If after trying every $T \subseteq[t]$ of size at most $2 \delta$, no tuple realizing $\left(H^{\prime}, H_{*}^{\prime}\right)$ is found, then by Proposition 4.11 we know that there is no tuple realizing $\left(H^{\prime}, H_{*}^{\prime}\right)$.

In Case $2(|U|<h)$, our algorithm for FINDIRRELEVANTORSEPARATION determines the $\delta$-folio of $G^{\prime}=G+X$ the following way. For every candidate $H$ in the $\delta$-folio, we enumerate every scheme $\left(H^{\prime}, H_{*}^{\prime}\right)$ of $H$ with $|V(H)| \leq 4 \delta+2 \delta^{2}$ (the number of such schemes is clearly bounded by a function of $\delta$ ). For each scheme, we use Lemma 4.13 to check if there is a tuple of clusters that realizes this scheme. If there is such a tuple, then by Lemma 4.10, we can obtain a model of $H$ in $G^{\prime}$ or a small separation; if there is no such tuple, then the (contrapositive) of Lemma 4.8 shows that $H^{\prime}$ is not a topological minor of $G^{\prime}$. It is easy to verify that for fixed $\delta$ and $L$, the running time is $O\left(|V(G)|^{2}\right)$. 


\section{Immersion}

Let $G, H$ be graphs. An immersion of $H$ in $G$ is a function $\alpha$ with domain $V(H) \cup E(H)$, such that:

- $\alpha(v) \in V(G)$ for all $v \in V(H)$, and $\alpha(u) \neq \alpha(v)$ for all distinct $u, v \in V(H)$,

- for each edge $e$ of $H$, if $e$ has distinct ends $u, v$ then $\alpha(e)$ is a path of $G$ with ends $\alpha(u), \alpha(v)$, and

- for all distinct $e, f \in E(H), E(\alpha(e) \cap \alpha(f))=\emptyset$.

In the definition of strong immersion, we impose on another condition, that

- for all $v \in V(H)$ and $e \in E(H)$, if $e$ is not incident with $v$ in $H$ then $\alpha(v) \notin V(\alpha(e))$.

In this section, we show that our main theorem, Theorem 1.1 implies that both versions of the immersion containment problem are fixed-parameter tractable parameterized by the order of $|E(H)|$.

THEOREM 5.1. For every fixed graph $H$, there is a $O\left(|V(G)|^{3}\right)$ time algorithm that decides if $H$ is an immersion in $G$.

Proof. Let $k=|E(H)|+|V(H)|$. We construct a new graph $G^{\prime}$ from $G$ by subdividing each edge and replacing each original vertex by $k$ duplicates. Formally, for each $e \in E(G)$, there is a vertex $e^{\prime}$ in $G^{\prime}$; for each vertex $v \in V(G)$, there are $k$ vertices $v_{1}, \ldots, v_{k}$ in $G^{\prime}$, and if $v \in V(G)$ is an endpoint of $e \in E(G)$, then vertex $e^{\prime} \in V\left(G^{\prime}\right)$ is adjacent to $v_{1}, \ldots, v_{k}$ in $G^{\prime}$. Note that the degree of $e^{\prime}$ is $2 k$.

Let $\ell=2 k|V(H)|+2$ and let us use the algorithm of Theorem 1.1 to find a $K_{\ell}$ topological minor in $G^{\prime}$. We claim that if there is such a topological minor model $\phi: V\left(K_{\ell}\right) \rightarrow V\left(G^{\prime}\right)$, then $H$ has an immersion in $G$. To see this, observe first that $\phi(v)$ is a vertex with degree at least $\ell-1>2 k$, thus $\phi(v)=u_{i}$ for some $u \in V(G)$; let us define $\alpha(v)=u$ in this case. It is clear that $\alpha$ maps at most $k$ vertices of $K_{\ell}$ to the same vertex of $G$. As $\ell / k>|V(H)|$ holds, one can select vertices $x_{1}, \ldots, x_{|V(H)|}$ whose images in $\phi$ are all distinct. For any $1 \leq i, j \leq|V(H)|$, the path $\phi\left(x_{i} x_{j}\right)$ between $\phi\left(x_{i}\right)$ and $\phi\left(x_{j}\right)$ in $G^{\prime}$ gives a path $\alpha\left(x_{i} x_{j}\right)$ between $\alpha\left(x_{i}\right)$ and $\alpha\left(x_{j}\right)$ in a natural way. As the paths $\phi\left(x_{i} x_{j}\right)$ are pairwise internally vertex disjoint in $G^{\prime}$, the paths $\alpha\left(x_{i} x_{j}\right)$ are pairwise edge disjoint in $G$ : a vertex $e^{\prime} \in E\left(G^{\prime}\right)$ can be used by at most one of the paths $\phi\left(x_{i} x_{j}\right)$. Therefore, $\phi$ shows that $K_{|V(H)|}$ has an immersion in $G$, which immediately implies that $H$ has an immersion in $G$. This means that we are done in the case when $K_{\ell}$ is a topological minor of $G^{\prime}$.

Suppose now that $K_{\ell}$ is not a topological minor of $G^{\prime}$. We modify $G^{\prime}$ to obtain a new graph $G^{\prime \prime}$ as follows. For every $v \in V(G)$, we introduce a new copy of $K_{\ell}$ and identify $v_{1}$ with a vertex of $K_{\ell}$. Thus the number of vertices of $G^{\prime \prime}$ is $\left|V\left(G^{\prime}\right)\right|+|V(G)|(\ell-1)$. Similarly, we obtain $H^{\prime \prime}$ from $H$ by introducing for each $u \in V(H)$ a new copy of $K_{\ell}$ and identifying $u$ and a vertex of $K_{\ell}$ (so $\left|V\left(H^{\prime \prime}\right)\right|=\ell|V(H)|$ ).

We claim that $H^{\prime \prime}$ is a topological minor of $G^{\prime \prime}$ if and only if $H$ has an immersion in $G$. For the if part, suppose that $\alpha$ is an immersion of $H$ in $G$. In this case, it is easy to construct a model $\phi$ of $H^{\prime \prime}$ in $G^{\prime \prime}$ : if $\alpha(u)=v$ for some $u \in V(H)$ and $v \in V(G)$, then we set $\phi(u)=v_{1}$, map the clique attached to $v$ in $H^{\prime \prime}$ to the clique attached to $v_{1}$, and transform each path $\alpha\left(u_{1} u_{2}\right)$ in $G$ into a corresponding path $\phi\left(u_{1} u_{2}\right)$ in $G^{\prime \prime}$. We can ensure that the paths in $\phi$ are internally vertex disjoint: the paths in $\alpha$ are edge disjoint (so we can ensure that each vertex $e^{\prime} \in V\left(G^{\prime \prime}\right)$ is used at most once) and the $k$ vertices $v_{1}, \ldots, v_{k}$ in $G^{\prime \prime}$ are sufficient to accommodate the at most $|E(H)|$ paths going through $v$ in $\alpha$.

For the only if part, suppose that $\phi$ is a model of $H^{\prime \prime}$ in $G^{\prime \prime}$. Consider a vertex $u$ of $H^{\prime \prime}$ that also appears in $H$ (i.e., it is not a vertex introduced by a new clique). The degree of $u$ in $H^{\prime \prime}$ is more than $\ell-1$ (assuming that $H$ has no isolated vertices) and $u$ is part of an $\ell$-clique in $H^{\prime \prime}$. Thus $\phi(u)$ is a vertex of $G^{\prime \prime}$ having degree more than $\ell-1$ and part of a topological minor model of a $\ell$-clique. We claim that $\phi(u)=v_{1}$ for some $v \in V(G)$. Every model of an $\ell$ clique is fully contained in a biconnected component of $G^{\prime \prime}$. As $G^{\prime}$ has no $\ell$-clique topological minor, such a biconnected component must be one of the $K_{\ell}$-cliques created in the construction of $G^{\prime \prime}$. Furthermore, the new vertices of such a clique have degree exactly $\ell-1$, thus $\phi(u)$ can be only a vertex $v_{1}$ for some $v \in V(G)$. Thus $\phi$ restricted to $H$ is a topological minor model of $H$ that does not go inside the cliques, which means that it is a topological minor model of $H$ in $G^{\prime}$. Arguing as in the first part of the proof, it follows that $H$ has an immersion in $G$.

Let us estimate the running time of the algorithm. First, we can assume that $|E(G)| \leq c_{H}|V(G)|$ for some constant $c_{H}$ depending only on $H$ : by a classical result of Mader, if the average degree of $G$ is sufficiently large, then $G$ has a $K_{|V(H)|}$ topological minor, immediately implying that $H$ has an immersion in $G$. Therefore, the number of vertices of $G^{\prime}$ is $k|V(G)|+|E(G)|=O(|V(G)|$ ) (for fixed $H$ ). The construction of $G^{\prime \prime}$ increases the number of vertices by a factor of $\ell$, hence $\left|V\left(G^{\prime \prime}\right)\right|=O(|V(G)|)$ also holds. Thus both invocations of Theorem 1.1 need $O\left(|V(G)|^{3}\right)$ time.

A similar reduction works in the case of strong immersion:

THEOREM 5.2. For every fixed graph $H$, there is a $O\left(|V(G)|^{3}\right)$ time algorithm that decides if $H$ is a strong immersion in $G$.

\section{References}

[1] S. Arnborg and A. Proskurowski. Linear time algorithms for NP-hard problems restricted to partial k-trees. Discrete Applied Mathematics, 23(1):11-24, 1989.

[2] H. L. Bodlaender. A linear-time algorithm for finding tree-decompositions of small treewidth. SIAM J. Comput., 25(6):1305-1317, 1996.

[3] R. G. Downey and M. R. Fellows. Fixed-parameter intractability. In Structure in Complexity Theory Conference, pages 36-49, 1992.

[4] R. G. Downey and M. R. Fellows. Parameterized Complexity. Monographs in Computer Science. Springer, New York, 1999.

[5] J. Flum and M. Grohe. Parameterized Complexity Theory. Springer, 2006.

[6] S. Fortune, J. E. Hopcroft, and J. Wyllie. The directed subgraph homeomorphism problem. Theor. Comput. Sci., 10:111-121, 1980.

[7] M. R. Garey and D. S. Johnson. Computers and Intractability. W. H. Freeman and Co., San Francisco, Calif., 1979.

[8] K. Kawarabayashi, Y. Kobayashi, and B. Reed. The disjoint paths problem in quaratic time. Submitted. Available at http://research.nii.ac.jp/ k_keniti/quaddp1.pdf.

[9] K. Kawarabayashi and P. Wollan. A shorter proof of the graph minor algorithm: the unique linkage theorem. In STOC, pages 687-694, 2010. A full version vailable at http://research.nii.ac.jp/ ${ }^{\sim}$ __keniti/uniquelink.pdf.

[10] A. S. LaPaugh and R. L. Rivest. The subgraph homeomorphism problem. J. Comput. Syst. Sci., 20(2):133-149, 1980.

[11] N. Robertson and P. D. Seymour. Graph minors. XIII. The disjoint paths problem. J. Combin. Theory Ser. B, 63(1):65-110, 1995.

[12] N. Robertson and P. D. Seymour. Graph minors. XVI. Excluding a non-planar graph. J. Comb. Theory, Ser. B, 89(1):43-76, 2003.

[13] N. Robertson and P. D. Seymour. Graph minors. XXI. Graphs with unique linkages. J. Comb. Theory, Ser. B, 99(3):583-616, 2009.

[14] N. Robertson and P. D. Seymour. Graph minors XXIII. nash-williams' immersion conjecture. J. Comb. Theory, Ser. B, 100(2):181-205, 2010.

[15] P. D. Seymour and R. Thomas. Graph searching and a min-max theorem for tree-width. J. Comb. Theory, Ser. B, 58(1):22-33, 1993.

[16] J. Thatcher and J. Wright. Generalised finite automata theory with an application to a decision problem of second-order logic. Mathematical Systems Theory, 2:57-81, 1968. 